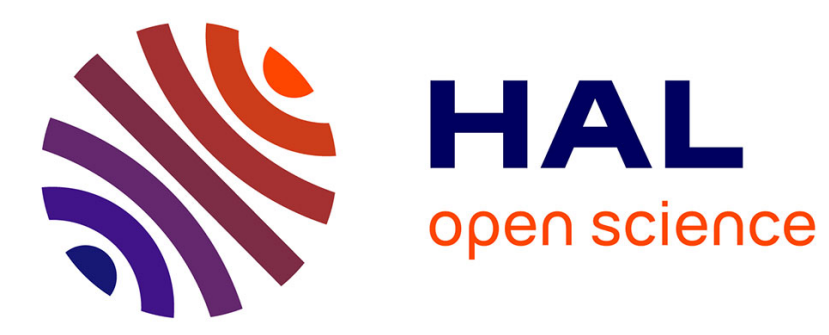

\title{
Colour texture classification from colour filter array images using various colour spaces
}

\author{
Olivier Losson, Ludovic Macaire
}

\section{To cite this version:}

Olivier Losson, Ludovic Macaire. Colour texture classification from colour filter array images using various colour spaces. IET Image Processing, 2012, 6 (8), pp.1192-1204. 10.1049/iet-ipr.2011.0556 . hal-00732780

\section{HAL Id: hal-00732780 \\ https://hal.science/hal-00732780}

Submitted on 22 Jan 2018

HAL is a multi-disciplinary open access archive for the deposit and dissemination of scientific research documents, whether they are published or not. The documents may come from teaching and research institutions in France or abroad, or from public or private research centers.
L'archive ouverte pluridisciplinaire HAL, est destinée au dépôt et à la diffusion de documents scientifiques de niveau recherche, publiés ou non, émanant des établissements d'enseignement et de recherche français ou étrangers, des laboratoires publics ou privés. 


\title{
Colour texture classification from CFA images using various colour spaces
}

\author{
Olivier Losson and Ludovic Macaire \\ olivier.losson@univ-lille1.fr, ludovic.macaire@univ-lille1.fr \\ Laboratoire LAGIS - UMR CNRS 8219 \\ Université Lille 1 - Sciences et Technologies \\ Cité Scientifique - Bâtiment P2 \\ 59655 Villeneuve d'Ascq - FRANCE
}

Version: January 22, 2018

This paper focuses on the classification of colour textures acquired by single-sensor colour cameras. In such cameras, the Colour Filter Array (CFA) makes each photosensor sensitive to only one colour component, and CFA images must be demosaiced to estimate the final colour images. We show that demosaicing is detrimental to the textural information because it affects colour texture descriptors such as Chromatic Co-occurrence Matrices (CCMs). However, it remains desirable to take advantage of the chromatic information for colour texture classification. This information is incompletely defined in CFA images, in which each pixel is associated to one single colour component. It is hence a challenge to extract standard colour texture descriptors from CFA images without demosaicing.

We propose to form a pair of quarter-size colour images directly from CFA images without any estimation, then to compute the CCMs of these quarter-size images. This allows us to compare textures by means of their CCM-based similarity in texture classification or retrieval schemes, with still the ability to use different colour spaces. Experimental results achieved on benchmark colour texture databases show the effectiveness of the proposed approach for texture classification, and a complexity study highlights its computational efficiency.

Key Words: Colour texture image, CFA demosaicing, Texture classification, Texture retrieval, Chromatic co-occurrence matrix, Colour space.

\section{INTRODUCTION}

Closely related to appearance-based object recognition, the problem of texture classification is a major field of low-level vision research. It is associated to various kinds of applications in visual inspection [1, 2, 3, 4], in medical [5], satellite [6], or multi-spectral $[7,8]$ image analysis, among many others. It consists in grouping a set of images or regions that represent textures into classes, by means of their similarity . This is a particular case of the more general problem of texture retrieval, whose goal is to retrieve the most similar target images to an input test image.

For this purpose, the textures are characterized by descriptors that are compared by means of a similarity measure. Among the various families of texture descriptors proposed in the literature, we focus on statistical descriptors, which are among the most investigated ones. Several experiments have indeed proved the efficiency of these descriptors in texture classification, due to their ability to describe any kind of texture. This family encompasses first-order (e.g., histograms) 
and second-order descriptors (e.g., local binary patterns [9], co-occurrence matrices $[10,11]$, interplane sum- and difference-histograms [7]), as well as descriptors of higher orders (e.g., run-length matrices [12]).

Palm [11] has shown that classification based on colour analysis outperforms that based on the luminance information only: colour information is worth being used together with texture information to serve as cues in a texture classifier. The colour characterizing each pixel is represented in a three-dimensional colour space (e.g., $R G B$ ) by a vector whose coordinates are colour component levels. Texture descriptors should then describe both the spatial arrangement of the colour component levels in the image plane and their distribution in a colour space. To characterize colour textures, we opt for the chromatic co-occurrence matrices introduced by Palm [11], since they are precisely able to capture spatial relationships within a single colour component and between different colour components.

Several studies (e.g., $[13,14])$ show that the choice of the colour space used to code colours impacts the texture classification accuracy. Hence, we need to carefully choose the colour space with respect to the application. However, these studies assume that colour information is well represented by the colour images, and neglect the impact of the acquisition device on texture characterization quality. Yet, colour images are often estimated by demosaicing from raw images (also known as Colour Filter Array (CFA) images), since the most widespread colour cameras are equipped with one single sensor [15]. Such colour estimation may degrade both the informational relevance of the texture descriptors and the relevance of the selected colour space, in comparison with images acquired by three-sensor cameras.

Several works deal with CFA data enhancement before demosaicing. For instance, the CFA image may be processed to improve rendering [16] or to remove noise $[17,18]$. But to our best knowledge, the literature reports no work that uses directly the CFA data to achieve texture classification. In this paper, we propose to avoid the demosaicing step and to use the colorimetric content of the CFA image for texture comparison. From this CFA image, we cannot directly represent colours in several colour spaces. So, we form new colour images without any demosaicing. These new images can be converted into different colour spaces. Then, they can be described by chromatic co-occurrence matrices for texture comparison.

The rest of the paper is organized as follows. First, we give some background information about texture comparison thanks to chromatic co-occurrence matrices. Then, we propose an original texture comparison scheme which analyses the CFA images acquired by single-sensor colour cameras. Finally, experimental results are presented to assess the relevance of our approach before conclusions are drawn.

\section{BACKGROUND OF COLOUR TEXTURE COMPARISON}

In this section, we first recall some properties of the various colour spaces that have been used in colour texture comparison. Then, we present how chromatic co-occurrence matrices are generally extracted from a colour image representing a texture. In the last subsection, we describe how these descriptors can be compared thanks to dedicated similarity measures. 


\subsection{Colour spaces}

Images acquired by three-sensor cameras are generally coded with the colour space $R G B$. Several other colour spaces exist, which are mostly defined by colorimetry. Considering various colour spaces is interesting, since each of them owns specific properties, and it is well-known that the choice of a given colour space impacts the accuracy of colour texture classification [13]. To study this influence, we propose to code the image colours in various spaces.

Let us quickly review the main existing colour spaces. Busin et al. [19] propose to group them into four families, with respect to their definitions and properties:

- The primary spaces are based on the trichromatic theory, according to which any colour can be formed by mixing an appropriate amount of three primary colours. The most used primary spaces are the $R G B$ ones, and the standard space of the International Commission on Illumination (CIE) $X Y Z$, based on imaginary (virtual) primary colours.

- The luminance-chrominance spaces are based on one colour component representing the luminance, and on two other components representing the chrominance. The main luminance-chrominance spaces are the television spaces $Y I Q$ and $Y U V$, and the CIE perceptually uniform spaces $L^{*} a^{*} b^{*}$ and $L^{*} u^{*} v^{*}$.

- The perceptual spaces, like $H S V$ and $H S L$, try to quantify the subjective human perception of colour by means of three measures: hue, saturation, and intensity or lightness.

- The Ohta colour space $I_{1} I_{2} I_{3}$ is a space of statistically-independent components resulting from the principal component analysis of the colours in several representative images.

To study the influence of the colour space on texture classification results, the colours of target and test images are coded in one of the above spaces before the texture descriptors are computed and compared.

\subsection{Chromatic co-occurrence matrices}

A Chromatic Co-occurrence Matrix (CCM) is a statistical texture descriptor that can be viewed as a generalization of the grey-level co-occurrence matrix proposed by Haralick [10]. It was introduced by Rosenfeld [20] and taken up by Palm [11] as a texture descriptor extracted from a colour image. Many studies have shown the efficiency of CCMs for colour texture classification (see [21], for instance). Indeed, these descriptors both take into account the colour distribution of an image and the spatial interactions between the colours of neighbouring pixels.

Let $\mathbf{I}^{\mathcal{E}}$ be a colour image whose colours are coded in the colour space $\mathcal{E}=$ $C_{1} C_{2} C_{3}$. This image is composed of three colour component images $I^{k}, k \in$ $\left\{C_{1}, C_{2}, C_{3}\right\}$, in which each pixel $P$ is characterized by the level $I^{k}(P)$ of the colour component $k$. The three-component vector $\mathbf{I}^{\mathcal{E}}(P)=\left(I^{C_{1}}(P), I^{C_{2}}(P), I^{C_{3}}(P)\right)^{T}$ is 
therefore associated with each pixel $P$. For computation efficiency reasons, the colour components are generally sub-quantized with $q$ levels before the CCMs are computed.

The CCM $M^{k, k^{\prime}}\left[\mathbf{I}^{\mathcal{E}}\right]$ captures the spatial interactions between the colour components $k$ and $k^{\prime}$ of the pixels in image $\mathbf{I}^{\mathcal{E}}$, according to a given neighbourhood $\mathcal{N}_{d}$. The cell $M^{k, k^{\prime}}\left[\mathbf{I}^{\mathcal{E}}\right](i, j)$ of this matrix, $0 \leq i<q, 0 \leq j<q$, contains the number of times that a pixel $Q$ whose level is $j$ for the colour component $k^{\prime}$, occurs in the neighbourhood $\mathcal{N}_{d}(P)$ of a pixel $P$ whose level is $i$ for the colour component $k$ :

$$
M^{k, k^{\prime}}\left[\mathbf{I}^{\mathcal{E}}\right](i, j)=\sum_{P \in \mathbf{I}^{\mathcal{E}}} \sum_{Q \in \mathcal{N}_{d}(P)} \begin{cases}1 & \text { if } I^{k}(P)=i \text { and } I^{k^{\prime}}(Q)=j, \\ 0 & \text { otherwise. }\end{cases}
$$

The neighbourhood $\mathcal{N}_{d}(P)$ used to compute the CCMs is made of the 8 pixels located at uniform distance (also called infinity-norm distance) equal to $d$ from $P$ along the four main directions of the image plane (horizontal and vertical directions, and the two diagonal ones):

$$
\mathcal{N}_{d}(P)=\left\{Q \in \mathbf{I}^{\mathcal{E}} \mid\|P Q\|_{\infty}=d, \theta=0(\bmod \pi / 4)\right\},
$$

where $\theta$ denotes the angle between the horizontal unit vector and the vector $P Q$ defined by the pixels $P$ and $Q$. The parameter $d$ is adjusted by the user to fit the texture granularity at best. Note that, even if different neighbourhoods can be considered [11], $\mathcal{N}_{d}$ is consistent with that proposed by Haralick [10].

As a measure of the local interaction between pixels computed from the whole image, a CCM is sensitive to any variation of the image size. To decrease this sensitivity, a CCM should be normalized by the total number of co-occurrences:

$$
m^{k, k^{\prime}}\left[\mathbf{I}^{\mathcal{E}}\right](i, j)=\frac{M^{k, k^{\prime}}\left[\mathbf{I}^{\mathcal{E}}\right](i, j)}{\sum_{a=0}^{q-1} \sum_{b=0}^{q-1} M^{k, k^{\prime}}\left[\mathbf{I}^{\mathcal{E}}\right](a, b)},
$$

so that all the cells of this normalized CCM $m^{k, k^{\prime}}\left[\mathbf{I}^{\mathcal{E}}\right]$ sum up to 1 . In the sequel, we always refer to normalized CCMs.

The relationships between the levels of neighbouring pixels within any of the three colour component images $I^{k}$ are represented by a single-component CCM $m^{k, k}\left[\mathbf{I}^{\mathcal{E}}\right], k \in\left\{C_{1}, C_{2}, C_{3}\right\}$. In order to provide information about the spatial relationships between the colour components, multi-component matrices (namely $m^{k, k^{\prime}}\left[\mathbf{I}^{\mathcal{E}}\right], k \neq k^{\prime}$ ) must be considered [11]. These matrices contain the number of pairwise occurrences of levels between different colour components. Because $m^{k, k^{\prime}}\left[\mathbf{I}^{\mathcal{E}}\right]$ and $m^{k^{\prime}, k}\left[\mathbf{I}^{\mathcal{E}}\right]$ are transpose of each other, these matrices contain the same information, and one of them is enough to describe the co-occurrence information between the colour components $k$ and $k^{\prime}$.

For a given colour image $\mathbf{I}^{\mathcal{E}}$ and a given neighbourhood $\mathcal{N}_{d}$, six CCMs are thus computed:

- three single-component matrices $m^{C_{1}, C_{1}}\left[\mathbf{I}^{\mathcal{E}}\right], m^{C_{2}, C_{2}}\left[\mathbf{I}^{\mathcal{E}}\right]$, and $m^{C_{3}, C_{3}}\left[\mathbf{I}^{\mathcal{E}}\right]$, 
- three multi-component matrices $m^{C_{1}, C_{2}}\left[\mathbf{I}^{\mathcal{E}}\right], m^{C_{1}, C_{3}}\left[\mathbf{I}^{\mathcal{E}}\right]$ and $m^{C_{2}, C_{3}}\left[\mathbf{I}^{\mathcal{E}}\right]$,

otherwise denoted as $m^{k, k^{\prime}}\left[\mathbf{I}^{\mathcal{E}}\right],\left(k, k^{\prime}\right) \in \mathcal{S}^{\mathcal{E}}=\left\{\left(C_{1}, C_{1}\right),\left(C_{2}, C_{2}\right),\left(C_{3}, C_{3}\right),\left(C_{1}, C_{2}\right)\right.$, $\left.\left(C_{1}, C_{3}\right),\left(C_{2}, C_{3}\right)\right\}$.

\subsection{Comparison of colour textures}

Matching a test image $\mathbf{I}_{\text {test }}^{\mathcal{E}}$ with a target image $\mathbf{I}_{\text {tar }}^{\mathcal{E}}$ is based on a pairwise comparison between their respective CCMs $m^{k, k^{\prime}}\left[\mathbf{I}_{\text {test }}^{\mathcal{E}}\right]$ and $m^{k, k^{\prime}}\left[\mathbf{I}_{\text {tar }}^{\mathcal{E}}\right],\left(k, k^{\prime}\right) \in \mathcal{S}^{\mathcal{E}}$. Any of the measures examined by Rubner et al. [22] to evaluate the similarity between histograms can be used to compare CCMs. Indeed, normalized CCMs can be considered as 2D joint histograms that estimate the joint probability density function underlying texture patterns [23]. Empirical studies [22, 24] show that $\chi^{2}$, Jeffrey Divergence $(J D)$ and $\mathcal{L}_{1}$ measures exhibit very similar performance to compare textures with multidimensional histograms. The Earth Mover's Distance $(E M D)$ is a cross-cell distance with interesting properties, and is recognized as the best similarity measure $[22,25]$. We do not retain it here mainly because its performance in texture classification drastically decreases as the number of cells increases [22]. Hence, we retain two similarity measures for our experimental tests (see section 4): the intersection between CCMs (that is one of the simplest similarity measures) [26] and the Jeffrey divergence between CCMs (that is one of the most efficient to compare textures) [22].

The intersection between two CCMs, for any pair of colour components $\left(k, k^{\prime}\right) \in$ $\mathcal{S}^{\mathcal{E}}$, is expressed as:

$$
\operatorname{Inter}\left(m^{k, k^{\prime}}\left[\mathbf{I}_{\text {test }}^{\mathcal{E}}\right], m^{k, k^{\prime}}\left[\mathbf{I}_{\text {tar }}^{\mathcal{E}}\right]\right)=\sum_{i=0}^{q-1} \sum_{j=0}^{q-1} \min \left(m^{k, k^{\prime}}\left[\mathbf{I}_{\text {test }}^{\mathcal{E}}\right](i, j), m^{k, k^{\prime}}\left[\mathbf{I}_{\text {tar }}^{\mathcal{E}}\right](i, j)\right) .
$$

The similarity measure between the test and target images is the mean of their pairwise CCM intersections [27]:

$$
\operatorname{SIM} M_{I}\left(\mathbf{I}_{\text {test }}^{\mathcal{E}}, \mathbf{I}_{\text {tar }}^{\mathcal{E}}\right)=\frac{1}{6} \sum_{\left(k, k^{\prime}\right) \in \mathcal{S}^{\mathcal{E}}} \text { Inter }\left(m^{k, k^{\prime}}\left[\mathbf{I}_{\text {test }}^{\mathcal{E}}\right], m^{k, k^{\prime}}\left[\mathbf{I}_{\text {tar }}^{\mathcal{E}}\right]\right) .
$$

When the two images $\mathbf{I}_{\text {test }}^{\mathcal{E}}$ and $\mathbf{I}_{\text {tar }}^{\mathcal{E}}$ share a similar spatial arrangement of colours, their similarity measure value $S I M_{I}$ is close to 1 . Although it does not necessarily mean that the two images contain the same texture, we assume so. On the other hand, a similarity measure value close to 0 means that the two textures are significantly different.

The Jeffrey divergence is derived from the Kullback-Leibler Divergence $(K L D)$. Both can be regarded as agreement measures between two probability density functions. In contrast to $K L D, J D$ is symmetric and presents a better numerical behaviour [22]: it is stable when comparing two empirical distributions. The Jeffrey divergence is expressed as: 


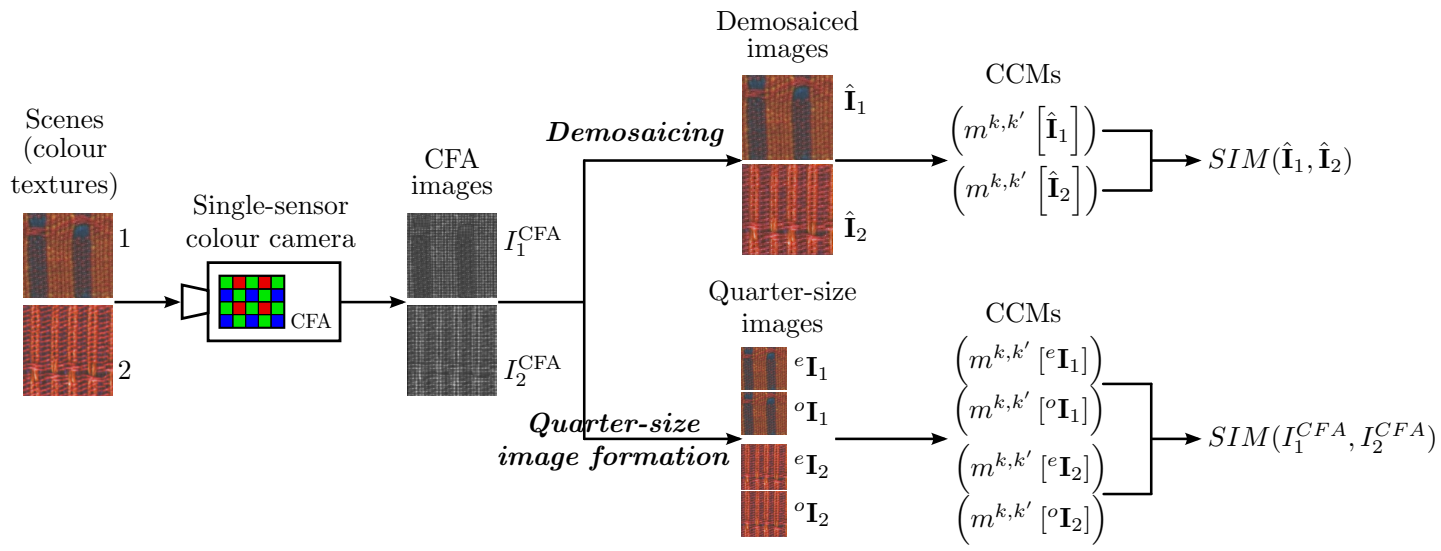

FIG. 1 Classical (top branch) and proposed (bottom branch) approaches for CCMbased comparison of colour textures.

$$
\begin{aligned}
J D\left(m^{k, k^{\prime}}\left[\mathbf{I}_{\text {test }}^{\mathcal{E}}\right], m^{k, k^{\prime}}\left[\mathbf{I}_{\text {tar }}^{\mathcal{E}}\right]\right)=\sum_{i=0}^{q-1} \sum_{j=0}^{q-1} & {\left[m^{k, k^{\prime}}\left[\mathbf{I}_{\text {test }}^{\mathcal{E}}\right](i, j) \times \log \left(\frac{m^{k, k^{\prime}}\left[\mathbf{I}_{\text {test }}^{\mathcal{E}}\right](i, j)}{\bar{m}^{k, k^{\prime}}(i, j)}\right)\right.} \\
& \left.+m^{k, k^{\prime}}\left[\mathbf{I}_{\text {tar }}^{\mathcal{E}}\right](i, j) \times \log \left(\frac{m^{k, k^{\prime}}\left[\mathbf{I}_{\text {tar }}^{\mathcal{E}}\right](i, j)}{\bar{m}^{k, k^{\prime}}(i, j)}\right)\right],
\end{aligned}
$$

where $\bar{m}^{k, k^{\prime}}(i, j)=\frac{1}{2}\left(m^{k, k^{\prime}}\left[\mathbf{I}_{\text {test }}^{\mathcal{E}}\right](i, j)+m^{k, k^{\prime}}\left[\mathbf{I}_{\text {tar }}^{\mathcal{E}}\right](i, j)\right)$. When the two images contain the same texture, the Jeffrey divergence between their CCMs is close to 0 , whereas it tends to infinity when they are quite different. The similarity between the two texture images is then defined as:

$$
S I M_{J}\left(\mathbf{I}_{\text {test }}^{\mathcal{E}}, \mathbf{I}_{\text {tar }}^{\mathcal{E}}\right)=\frac{1}{1+\frac{1}{6} \sum_{\left(k, k^{\prime}\right) \in \mathcal{S}^{\mathcal{E}}} J D\left(m^{k, k^{\prime}}\left[\mathbf{I}_{\text {test }}^{\mathcal{E}}\right], m^{k, k^{\prime}}\left[\mathbf{I}_{\text {tar }}^{\mathcal{E}}\right]\right)}
$$

\section{COLOUR TEXTURE COMPARISON FROM CFA IMAGES}

Using various colour spaces for texture comparison requires that colours and textures are well represented in images. To ensure this at best, colour images should be acquired by three-sensor colour cameras, in which each sensor is associated with a colour component $R, G$, or $B$. However, most colour texture images are presently acquired by colour cameras equipped with one single sensor. This sensor acquires CFA images from which colour images are estimated by demosaicing, as generically described in the first subsection below.

To compare two colour textures, classical approaches compute the CCMs of demosaiced images, then estimate the texture similarity according to equation (5) 
or (7) (see figure 1, top branch). In the second subsection, we experimentally show that colour estimation by demosaicing strongly alters the CCMs, which may decrease the performance of CCM-based texture classification. To avoid this, we propose to analyse CFA images in two steps (see figure 1, bottom branch). Two quarter-size colour images are directly formed from a CFA image (see third subsection). The fourth subsection describes how to compute CCMs from these quartersize images for colour texture comparison, with no demosaicing step and still the ability to use different colour spaces.

\subsection{CFA Image and demosaicing}

A colour camera fitted with a single sensor (either CCD or CMOS) incorporates a colour filter array, which makes each photosensor mainly sensitive to a specific primary colour. As a result, such a camera forms a CFA image (denoted as $I^{\mathrm{CFA}}$ ), in which a single colour component $R, G$, or $B$, is associated to each pixel. We consider here the most widespread CFA, namely the Bayer CFA. It yields the image $I^{\mathrm{CFA}}$ of figure 2(a), where the notations $R_{x, y}, G_{x, y}$, and $B_{x, y}$, express that the respective colour component is available at the pixel of spatial coordinates $(x, y), 0 \leq x<X$, $0 \leq y<Y$. According to the parity of these spatial coordinates, the single colour component available at pixel $(x, y)$ in $I^{\mathrm{CFA}}$ is:

- the $R$ colour component if $x$ is odd and $y$ is even,

- the $G$ colour component if $x$ and $y$ are of same parity,

- the $B$ colour component if $x$ is even and $y$ is odd.

To obtain a colour image from $I^{\mathrm{CFA}}$, a process called demosaicing is applied. Presenting demosaicing procedures is beyond the scope of this paper, and the reader may refer to [28] or [15] for a review; we only explain their common basic principle below.

To determine the colour $\left(\hat{I}^{R}(x, y), \hat{I}^{G}(x, y), \hat{I}^{B}(x, y)\right)^{T}$ of each pixel $P(x, y)$ in the demosaiced colour image $\hat{\mathbf{I}}^{R G B}$, the demosaicing process (denoted as $\mathcal{D}$ ) generally retains the colour component available at the same location in $I^{\mathrm{CFA}}$, and estimates the two missing colour components:

$$
I^{\mathrm{CFA}}(x, y) \stackrel{\mathcal{D}}{\rightarrow} \hat{\mathbf{I}}^{R G B}(x, y)= \begin{cases}\left(I^{\mathrm{CFA}}(x, y), \hat{I}^{G}(x, y), \hat{I}^{B}(x, y)\right)^{T} & \text { if } x \text { is odd and } y \text { is even }, \\ \left(\hat{I}^{R}(x, y), I^{\mathrm{CFA}}(x, y), \hat{I}^{B}(x, y)\right)^{T} & \text { if } x \text { and } y \text { are of same parity } \\ \left(\hat{I}^{R}(x, y), \hat{I}^{G}(x, y), I^{\mathrm{CFA}}(x, y)\right)^{T} & \text { if } x \text { is even and } y \text { is odd. }\end{cases}
$$

Each colour component triplet in equation (8) represents an estimated colour. Out of the three components in $\hat{\mathbf{I}}^{R G B}(x, y)$, the one available in $I^{\mathrm{CFA}}$ is denoted as $I^{\mathrm{CFA}}(x, y)$, and the missing other two components among $\hat{I}^{R}(x, y), \hat{I}^{G}(x, y)$, and $\hat{I}^{B}(x, y)$, are estimated by demosaicing. 


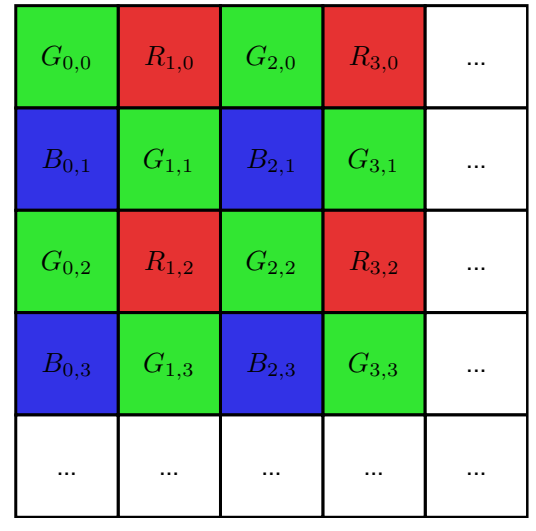

(a) CFA image $I^{\text {CFA }}$

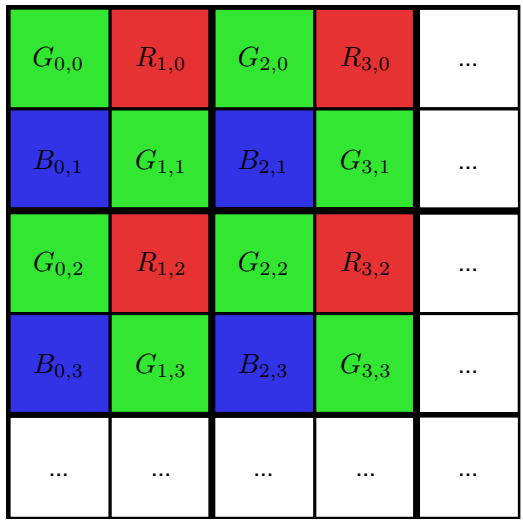

(b) CFA image blocks

FIG. 2 CFA image from the Bayer colour filter array, and its $2 \times 2$ block tile. Each pixel is artificially coloured with the main spectral sensitivity of the corresponding selective filter.

\subsection{CCM fidelity of demosaiced images}

The evaluation of the demosaicing performance generally uses an original colour image, from which the CFA image is "simulated" by sampling a single colour component at each pixel according to the Bayer CFA arrangement of figure 2(a). The considered demosaicing procedure is applied to this CFA image, and the demosaicing performance is evaluated as a fidelity measure between the demosaiced colour image and the original one. However, classical fidelity measures, as the Peak Signalto-Noise Ratio $(P S N R)$, use pixel-to-pixel colour comparison. So, they may poorly reflect to which extent the demosaicing procedure affects texture descriptors that provide information about the relationships between colours of neighbouring pixels. To quantify how demosaicing specifically degrades the CCMs, we propose to compare the CCMs computed from the demosaiced image and those directly computed from the original image.

Figure 3 outlines the procedure set up to achieve this comparison. We use the original colour images of the VisTex texture database [29]. Each original colour image $\mathbf{I}^{R G B}$ is uniformly quantized with $q$ levels (see subsection 4.1 for details) to obtain $\mathbf{I}_{(q)}^{R G B}$, from which six CCMs are computed as described in subsection 2.2. Each original colour image $\mathbf{I}^{R G B}$ is also sampled to produce the simulated CFA image $I^{\mathrm{CFA}}$. The CCMs are computed from the demosaiced colour image $\hat{\mathbf{I}}^{R G B}$, obtained by applying a highly-efficient demosaicing procedure to the simulated CFA image. Finally, the normalized CCMs computed from $\mathbf{I}_{(q)}^{R G B}$ and $\hat{\mathbf{I}}_{(q)}^{R G B}$ are compared using the intersection-based similarity of these images (see equation (5)). A similarity value close to 1 means that the demosaicing step does not strongly alter the CCMs, in comparison with those extracted from the original colour image.

Table 1 displays the comparison results obtained on 432 images from the Vis- 


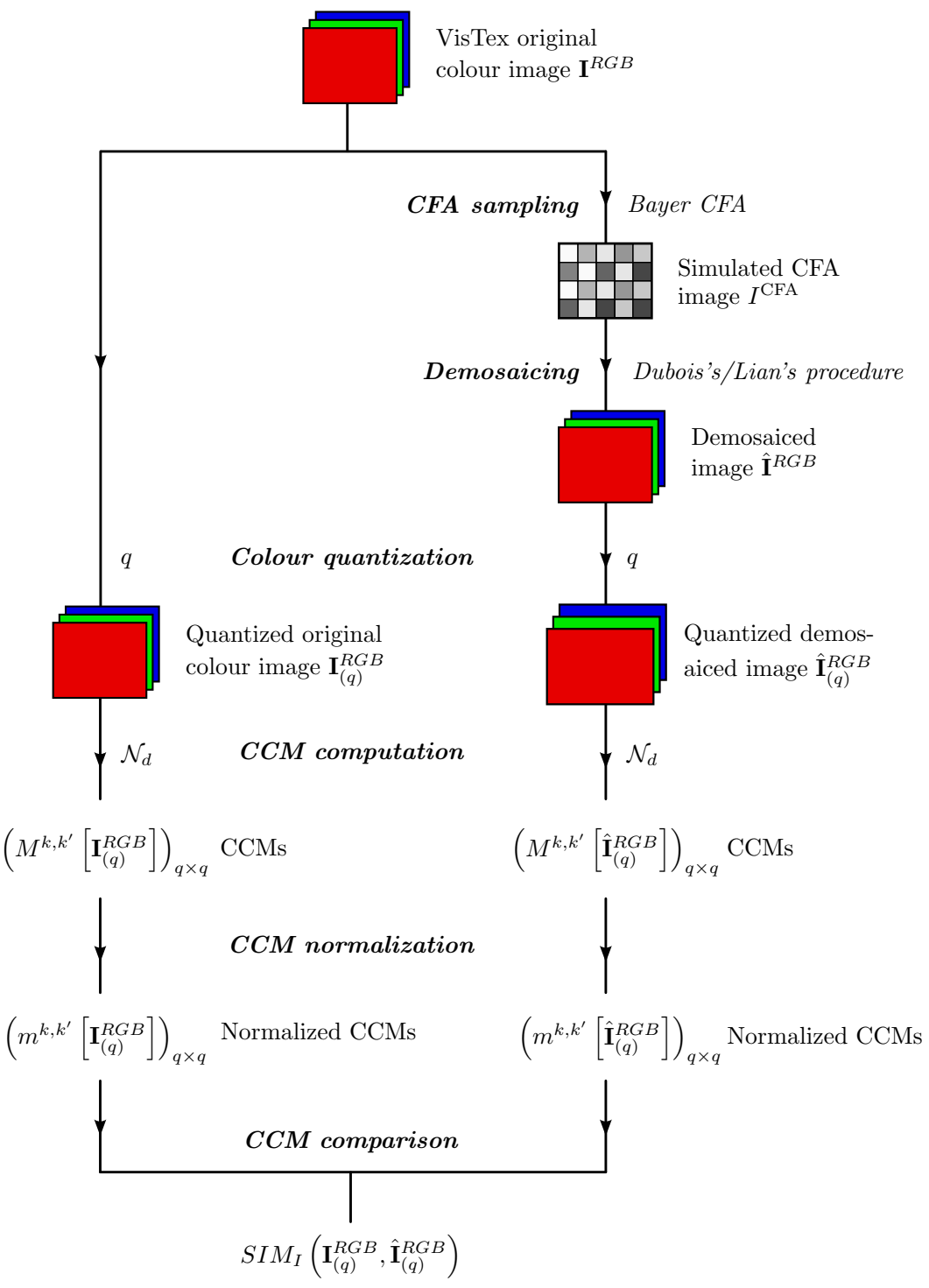

FIG. 3 Comparison between the CCMs computed from the original colour image $\mathbf{I}^{R G B}$ (left branch) and from the corresponding demosaiced image $\hat{\mathbf{I}}^{R G B}$ (right branch). 
TABLE 1: Intersection-based CCM similarity between the original colour image $\mathbf{I}_{(q)}^{R G B}$ and the demosaiced image $\hat{\mathbf{I}}_{(q)}^{R G B}$. The values indicate the means and standard deviations $(\mu \pm \sigma)$, when the distance parameter $d$ is set to 2 or 8 , for two demosaicing procedures and five values of the quantization level $q$.

\begin{tabular}{|r||c|c||c|c|}
\hline \multicolumn{1}{|c||}{} & \multicolumn{2}{c||}{$d=2$} & \multicolumn{2}{c|}{$d=8$} \\
$q$ & Dubois & Lian & Dubois & Lian \\
\hline 16 & $0.96 \pm 0.83 \times 10^{-3}$ & $0.96 \pm 0.63 \times 10^{-3}$ & $0.96 \pm 0.83 \times 10^{-3}$ & $0.96 \pm 0.64 \times 10^{-3}$ \\
32 & $0.94 \pm 1.00 \times 10^{-3}$ & $0.94 \pm 0.81 \times 10^{-3}$ & $0.94 \pm 1.03 \times 10^{-3}$ & $0.94 \pm 0.85 \times 10^{-3}$ \\
64 & $0.90 \pm 1.27 \times 10^{-3}$ & $0.91 \pm 1.16 \times 10^{-3}$ & $0.90 \pm 1.36 \times 10^{-3}$ & $0.91 \pm 1.27 \times 10^{-3}$ \\
128 & $0.84 \pm 2.03 \times 10^{-3}$ & $0.84 \pm 2.00 \times 10^{-3}$ & $0.82 \pm 2.26 \times 10^{-3}$ & $0.83 \pm 2.23 \times 10^{-3}$ \\
256 & $0.71 \pm 3.74 \times 10^{-3}$ & $0.72 \pm 3.73 \times 10^{-3}$ & $0.69 \pm 4.04 \times 10^{-3}$ & $0.69 \pm 4.03 \times 10^{-3}$ \\
\hline
\end{tabular}

Tex database, for five values of the quantization level $q$, two values of the distance parameter $d$ (see equation (2)), and with Dubois's [30] and Lian et al.'s [31] demosaicing procedures that, in turn, provide the best results with respect to many fidelity measures [15]. This table shows that, whatever the demosaicing procedure and distance parameter value, CCMs computed from $\hat{\mathbf{I}}_{(q)}^{R G B}$ differ from those computed from $\mathbf{I}_{(q)}^{R G B}$. A difference of $4 \%$ can already be noticed with the very coarse quantization using $q=16$ levels that keeps only the four most significant bits of original levels. The difference is all the more significant than the colour components are finely quantized, and it reaches about $30 \%$ with the original quantization $(q=256)$. The very small values of standard deviations indicate that the mean similarity values are highly representative of the results. Besides, the distance parameter $d$ hardly influences the CCM fidelity for a given quantization level $q$. In the rest of the paper, this parameter is therefore fixed to $d=2$.

This study highlights that demosaicing seriously affects the CCM values and, subsequently, may degrade the results of CCM-based texture classification. When the classification scheme is applied to texture images acquired by single-sensor colour cameras, it is therefore desirable to avoid the demosaicing step. The following is an attempt to use CCMs as colour texture descriptors extracted from CFA images.

\subsection{Quarter-size colour images from a CFA image}

The Bayer CFA used to form the image of figure 2(a) has a basic pattern of size $2 \times 2$ that contains twice more green filters than red or blue ones. The corresponding CFA image may therefore be considered as a tile of disjoint $2 \times 2$ blocks, each of which contains two pixels providing a green level, one pixel providing a red level, and one pixel providing a blue level (see figure 2(b)). A CFA image $I^{\text {CFA }}$ of size $X \times Y$ pixels is tiled with $\frac{X}{2} \frac{Y}{2}$ blocks, each one $(u, v)$ being formed of the levels denoted as $G_{2 u, 2 v}, G_{2 u+1,2 v+1}, R_{2 u+1,2 v}$, and $B_{2 u, 2 v+1}$ in figure 2, with $0 \leq u<\frac{X}{2}$, $0 \leq v<\frac{Y}{2}$. 


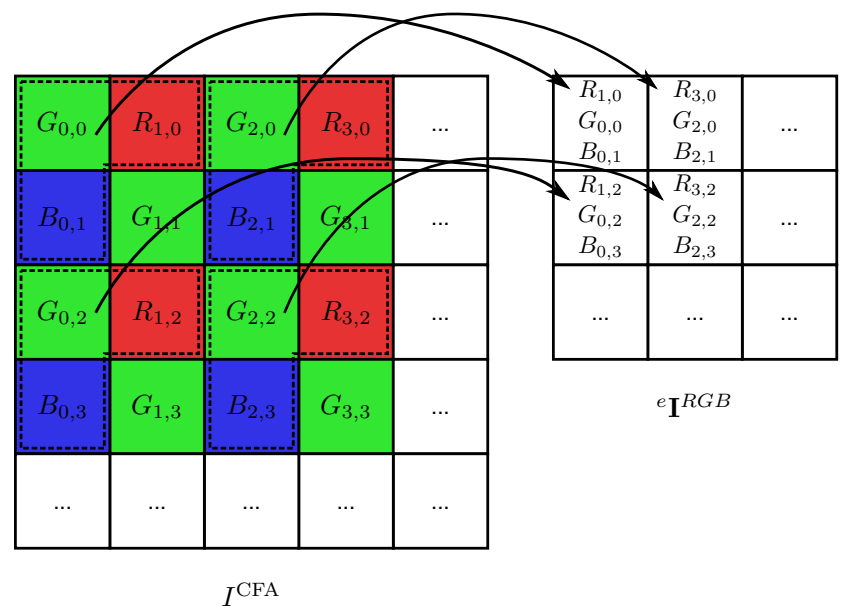

(a) Quarter-size colour image ${ }^{e} \mathbf{I}^{R G B}$

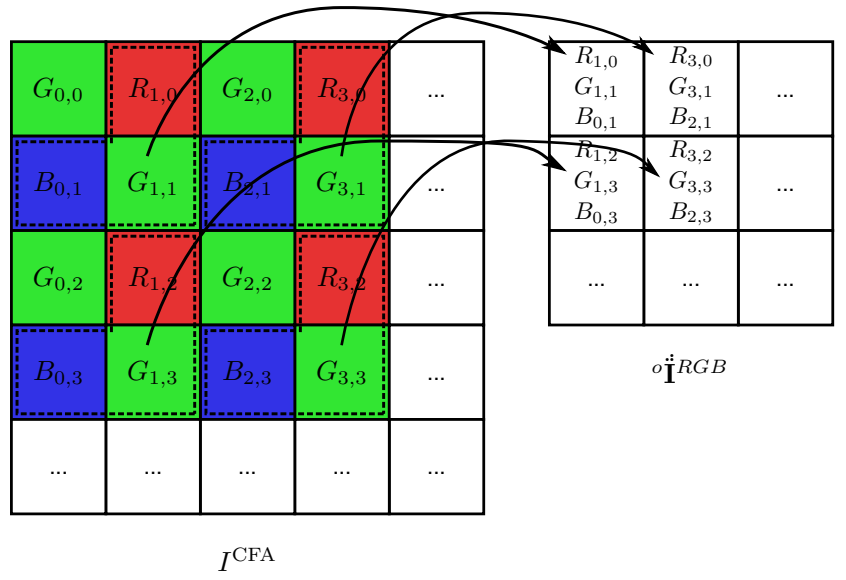

(b) Quarter-size colour image ${ }^{o} \dot{\mathbf{I}}^{R G B}$

FIG. 4 Formation of two quarter-size colour images from CFA image blocks. 
The colorimetric information contained in a $2 \times 2$ block can be used to characterize a colour. More precisely, the $R, G$, and $B$ levels in a block can be combined as the triplet of colour components to characterize the colour of a pixel in a $R G B$ colour image. Since a block contains two pixels where the green level is available in the CFA image, we can form two different colours from each block. Considering the global block tile, this allows us to form two different colour images from a given CFA image. These images are called quarter-size colour images, since their size is four times lower than that of the CFA image. The first quarter-size colour image is denoted as ${ }^{e} \mathbf{I}^{R G B}$, since it contains the green levels available at even spatial coordinates in $I^{\mathrm{CFA}}$. The second one is similarly denoted as ${ }^{o} \dot{\mathbf{I}}^{R G B}$, to recall that it is created from the green levels available at odd spatial coordinates (the extra diacritic above $\mathbf{I}$ is justified later). The colour of each pixel $(u, v)$ of these images, $0 \leq u<\frac{X}{2}, 0 \leq v<\frac{Y}{2}$, is defined in the $R G B$ colour space by (see figure 4 ):

$$
\begin{aligned}
& { }^{e} \mathbf{I}^{R G B}(u, v)=\left(I^{\mathrm{CFA}}(2 u+1,2 v), I^{\mathrm{CFA}}(2 u, 2 v), I^{\mathrm{CFA}}(2 u, 2 v+1)\right)^{T}, \\
& { }^{o} \ddot{\mathbf{I}}^{R G B}(u, v)=\left(I^{\mathrm{CFA}}(2 u+1,2 v), I^{\mathrm{CFA}}(2 u+1,2 v+1), I^{\mathrm{CFA}}(2 u, 2 v+1)\right)^{T} .
\end{aligned}
$$

In that way, from a CFA image $I^{\mathrm{CFA}}$, we form a pair of quarter-size colour images composed of an even image ${ }^{e} \mathbf{I}^{R G B}$ and an odd one ${ }^{o} \dot{\mathbf{I}}^{R G B}$.

The definitions of these two quarter-size colour images imply that the sole green channel differs between them (i.e., ${ }^{e} I^{G} \neq{ }^{\circ \tilde{I}^{G}}$ ), whereas the other two colour channels are identical (i.e., ${ }^{e} I^{R} \equiv{ }^{o} \dot{I}^{R}$ and ${ }^{e} I^{B} \equiv{ }^{o} \dot{I}^{B}$ ). However, considering the green level $I^{\mathrm{CFA}}(2 u+1,2 v+1)$ available at odd coordinates in the CFA image, various choices of $(R, G, B)$ triplets may form the colour of pixel $(u, v)$ in the odd quarter-size colour image (see figure 5 ). Indeed, both the upper and lower neighbouring pixels may provide the red level, and both the left and right neighbouring pixels may provide the blue level. The four resulting combinations yield four different possibilities for the odd quarter-size colour image - the even one ${ }^{e} \mathbf{I}^{R G B}$ being fixed, since the two quarter-size images in a pair provide complementary texture information. These four cases, depicted on figure 5 for the first pixel $(u=0, v=0)$, are denoted as ${ }^{o} \ddot{\mathbf{I}}^{R G B}$ (introduced above), ${ }^{o} \ddot{\mathbf{I}}^{R G B},{ }^{o} \ddot{\mathbf{I}}^{R G B}$, and ${ }^{o} \ddot{\mathbf{I}}^{R G B}$. They are respectively defined by equation (10) and by:

$$
\begin{aligned}
& { }^{o} \dot{\mathbf{I}}^{R G B}(u, v)=\left(I^{\mathrm{CFA}}(2 u+1,2 v), I^{\mathrm{CFA}}(2 u+1,2 v+1), I^{\mathrm{CFA}}(2 u+2,2 v+1)\right)^{T}, \\
& { }^{o} \ddot{\mathbf{I}}^{R G B}(u, v)=\left(I^{\mathrm{CFA}}(2 u+1,2 v+2), I^{\mathrm{CFA}}(2 u+1,2 v+1), I^{\mathrm{CFA}}(2 u+2,2 v+1)\right)^{T}, \\
& { }^{o} \ddot{\mathbf{I}}^{R G B}(u, v)=\left(I^{\mathrm{CFA}}(2 u+1,2 v+2), I^{\mathrm{CFA}}(2 u+1,2 v+1), I^{\mathrm{CFA}}(2 u, 2 v+1)\right)^{T} .
\end{aligned}
$$




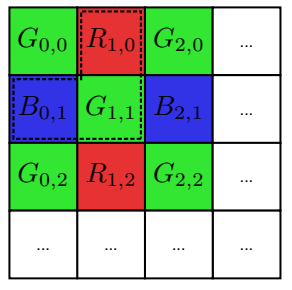

(a) ${ }^{o} \dot{\mathbf{I}}^{R G B}(0,0)$

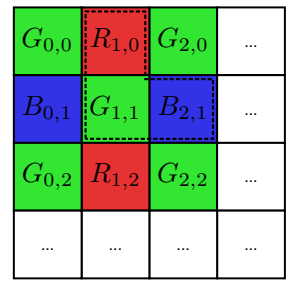

(b) ${ }^{o} \dot{\mathbf{I}^{R G B}}(0,0)$

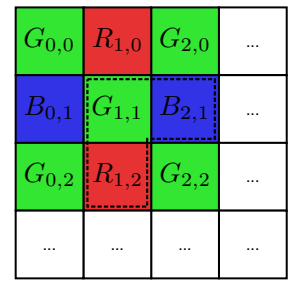

(c) ${ }^{o} \ddot{\mathbf{I}}^{R G B}(0,0)$

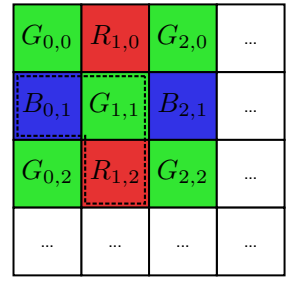

(d) ${ }^{\circ} \ddot{\mathbf{I}}^{R G B}(0,0)$

FIG. 5 The four possible $(R, G, B)$ triplets used to form the odd quarter-size colour image of figure 4(b) (illustration for the first pixel).

Although these last three cases do not strictly correspond any more to the " $2 \times 2$ block reasoning" used above to introduce ${ }^{o} \dot{\mathbf{I}}^{R G B}$, they may offer the advantage to provide more informative CCMs. Indeed, they contain less redundant information with ${ }^{e} \mathbf{I}^{R G B}$ than ${ }^{\circ} \dot{\mathbf{I}}^{R G B}$ : in addition to the green channel, the red channel of ${ }^{o} \ddot{\mathbf{I}}^{R G B}$ and ${ }^{o} \ddot{\mathbf{I}}^{R G B}$ differs from that of ${ }^{e} \mathbf{I}^{R G B}$, whereas the blue channel of ${ }^{o} \dot{\mathbf{I}}^{R G B}$ and ${ }^{o} \ddot{\mathbf{I}}^{R G B}$ differs from that of ${ }^{e} \mathbf{I}^{R G B}$.

In the following, the odd quarter-size colour image is denoted as ${ }^{o} \mathbf{I}^{R G B}$ in a generic way. The image actually used (either ${ }^{o} \dot{\mathbf{I}}^{R G B},{ }^{o} \dot{\mathbf{I}}^{R G B},{ }^{o} \ddot{\mathbf{I}}^{R G B}$, or ${ }^{o} \ddot{\mathbf{I}}^{R G B}$ ) to compute the CCMs from $I^{\mathrm{CFA}}$ is only explicitly specified when needed (e.g., when comparing the performances of these four cases for texture classification - see subsection 4.2).

Unlike demosaiced colour images, the quarter-size colour images ${ }^{e} \mathbf{I}^{R G B}$ and ${ }^{o} \mathbf{I}^{R G B}$ do not result from any colour estimation. But, on the other hand, their size is reduced in comparison with the CFA image. However, we assume that this pair of quarter-size colour images may be a relevant representation of the texture information embedded in the associated CFA image. Before this can be shown by experimental results, let us make clear how these images are used to compare colour textures.

\subsection{Comparison of colour textures from CFA images}

As explained above, the CFA image $I^{\mathrm{CFA}}$ has a the particular pattern, which hardly allows us to compute CCMs from it directly and to use other spaces than $R G B$ for colour coding. That is why we propose to form a pair of quarter-size colour images ${ }^{e} \mathbf{I}^{R G B}$ and ${ }^{o} \mathbf{I}^{R G B}$ from $I^{\mathrm{CFA}}$ (see equations (9) and (10)-(13)). Once their colours have been converted from the original $R G B$ space into the colour space $\mathcal{E}=C_{1} C_{2} C_{3}$, we compute CCMs from ${ }^{e} \mathbf{I}^{\mathcal{E}}$ and ${ }^{o} \mathbf{I}^{\mathcal{E}}$. To describe a texture represented by a given image $I^{\mathrm{CFA}}$, twelve normalized CCMs are thus computed: 
- six single-component matrices $m^{C_{1}, C_{1}}\left[{ }^{e} \mathbf{I}^{\mathcal{E}}\right], m^{C_{2}, C_{2}}\left[{ }^{e} \mathbf{I}^{\mathcal{E}}\right], m^{C_{3}, C_{3}}\left[{ }^{e} \mathbf{I}^{\mathcal{E}}\right], m^{C_{1}, C_{1}}\left[{ }^{o} \mathbf{I}^{\mathcal{E}}\right]$, $m^{C_{2}, C_{2}}\left[{ }^{o} \mathbf{I}^{\mathcal{E}}\right]$, and $m^{C_{3}, C_{3}}\left[{ }^{o} \mathbf{I}^{\mathcal{E}}\right]$.

- six multi-component matrices $m^{C_{1}, C_{2}}\left[{ }^{e} \mathbf{I}^{\mathcal{E}}\right], m^{C_{1}, C_{3}}\left[{ }^{e} \mathbf{I}^{\mathcal{E}}\right], m^{C_{2}, C_{3}}\left[{ }^{e} \mathbf{I}^{\mathcal{E}}\right], m^{C_{1}, C_{2}}\left[{ }^{o} \mathbf{I}^{\mathcal{E}}\right]$, $m^{C_{1}, C_{3}}\left[{ }^{o} \mathbf{I}^{\mathcal{E}}\right]$, and $m^{C_{2}, C_{3}}\left[{ }^{o} \mathbf{I}^{\mathcal{E}}\right]$.

Since twelve CCMs are now computed, the similarity measures presented in subsection 2.3 should be adjusted to compare two colour textures from their CFA images. The intersection-based similarity measure (see equation (5)) becomes:

$$
\begin{aligned}
& \operatorname{SIM}_{I}^{\mathcal{E}}\left(I_{\text {test }}^{\mathrm{CFA}}, I_{\text {tar }}^{\mathrm{CFA}}\right)= \\
& \frac{1}{12} \sum_{\left(k, k^{\prime}\right) \in \mathcal{S}^{\mathcal{E}}} \text { Inter }\left(m^{k, k^{\prime}}\left[{ }^{e} \mathbf{I}_{\text {test }}^{\mathcal{E}}\right], m^{k, k^{\prime}}\left[{ }^{e} \mathbf{I}_{\text {tar }}^{\mathcal{E}}\right]\right)+\operatorname{Inter}\left(m^{k, k^{\prime}}\left[{ }^{o} \mathbf{I}_{\text {test }}^{\mathcal{E}}\right], m^{k, k^{\prime}}\left[{ }^{o} \mathbf{I}_{\text {tar }}^{\mathcal{E}}\right]\right) .
\end{aligned}
$$

The similarity measure based on the Jeffrey divergence (see equation (7)) becomes:

$$
\begin{aligned}
& S I M_{J}^{\mathcal{E}}\left(I_{\text {test }}^{\mathrm{CFA}}, I_{\text {tar }}^{\mathrm{CFA}}\right)= \\
& \frac{1}{1+\frac{1}{12} \sum_{\left(k, k^{\prime}\right) \in \mathcal{S}^{\mathcal{E}}} J D\left(m^{k, k^{\prime}}\left[{ }^{e} \mathbf{I}_{\text {test }}^{\mathcal{E}}\right], m^{k, k^{\prime}}\left[{ }^{e} \mathbf{I}_{\text {tar }}^{\mathcal{E}}\right]\right)+J D\left(m^{k, k^{\prime}}\left[{ }^{0} \mathbf{I}_{\text {test }}^{\mathcal{E}}\right], m^{k, k^{\prime}}\left[{ }^{o} \mathbf{I}_{\text {tar }}^{\mathcal{E}}\right]\right)} .
\end{aligned}
$$

Thanks to the proposed pair of quarter-size images, we can compare two CFA images that are acquired by single-sensor colour cameras, while using several colour spaces.

\section{EXPERIMENTS}

This section intends to assess the relevance of the proposed texture representation from CFA images. This new representation is used for texture comparison as described above, and is assessed here within two experimental schemes: colour texture classification and retrieval.

In the first subsection, we present the texture datasets used in these experiments. We also give an overview of the experimental scheme, and discuss its parameters of neighbourhood distance and colour component quantization. We then study which pair of quarter-size images, among the four possible ones presented in subsection 3.3, should be retained on classification performance bases. In the third subsection, we present detailed results of texture classification accuracy and texture retrieval performance. The fourth subsection gives some insights about the computational complexity of our texture comparison scheme. 

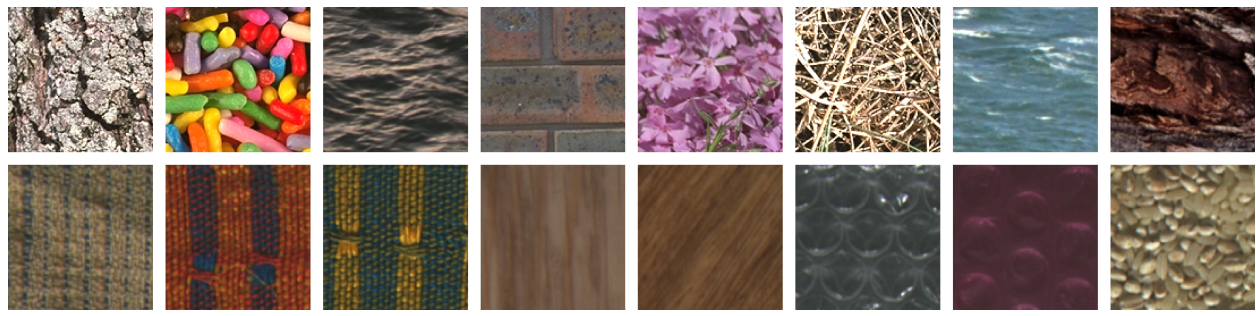

FIG. 6 Examples of VisTex (top row) and Outex (bottom row) colour textures.

\subsection{Experimental scheme}

For these experiments, we use two well-known colour texture databases, widely referred to as benchmarks: VisTex [29] and Outex [32]. The used datasets are the Contrib-TC-00006 and Outex-TC-00013 classification test suites, both available from the Outex web site [32]. The VisTex dataset contains 54 images of size $512 \times 512$ pixels, representing natural textured materials and objects (e.g., bark, clouds, flowers, grass, water) and acquired by various colour cameras under uncontrolled conditions. The Outex dataset contains 68 images of size $746 \times 538$ pixels, representing textured materials (e.g., granite, leather, plastic, seeds, wood) and acquired by a three-CCD colour camera under the same controlled conditions. All these RGB images are split into sub-images of size $128 \times 128$ pixels, which yields 16 (VisTex) and 20 (Outex) images for each texture class. The holdout procedure states that half of the images are the prototypes of the corresponding class, and half are used for the tests. Figure 6 illustrates 8 prototypes of different classes in each dataset.

The computation scheme of CCMs from these colour texture images is outlined in figure 7. We assume that the original colour images in the two datasets do not result from any demosaicing. The corresponding CFA images that a single-sensor camera would have provided are not available in the datasets. Thus, we practically simulate the CFA image $I^{\mathrm{CFA}}$ from its original colour image $\mathbf{I}^{R G B}$, as done in subsection 3.2. From $I^{\mathrm{CFA}}$, we apply a highly-efficient demosaicing procedure to compute the demosaiced colour image $\hat{\mathbf{I}}^{R G B}$. From $I^{\mathrm{CFA}}$, we also form the quartersize colour images ${ }^{e} \mathbf{I}^{R G B}$ and ${ }^{o} \mathbf{I}^{R G B}$ (see equations (9) and (10)-(13)).

The demosaiced and quarter-size images are then converted from $R G B$ into another colour space $\mathcal{E}$ to produce $\hat{\mathbf{I}}^{\mathcal{E}},{ }^{e} \mathbf{I}^{\mathcal{E}}$, and ${ }^{o} \mathbf{I}^{\mathcal{E}}$. The selected space is the one with which Mäenpää and Pietikäinen [26] obtain the highest accuracy of texture classification, namely Ohta colour space $I_{1} I_{2} I_{3}$ for VisTex images, and the perceptual colour space $H S V$ for Outex images.

Before computing the CCMs, we apply a uniform colour quantization [26] to code each colour component with $q$ levels only; this yields $\hat{\mathbf{I}}_{(q)}^{\mathcal{E}},{ }^{e} \mathbf{I}_{(q)}^{\mathcal{E}}$, and ${ }^{o} \mathbf{I}_{(q)}^{\mathcal{E}}$. Since each CCM computed from these quantized images contains only $q^{2}$ cells, this allows us to reduce its memory cost and to speed up its computation. Poreb- 


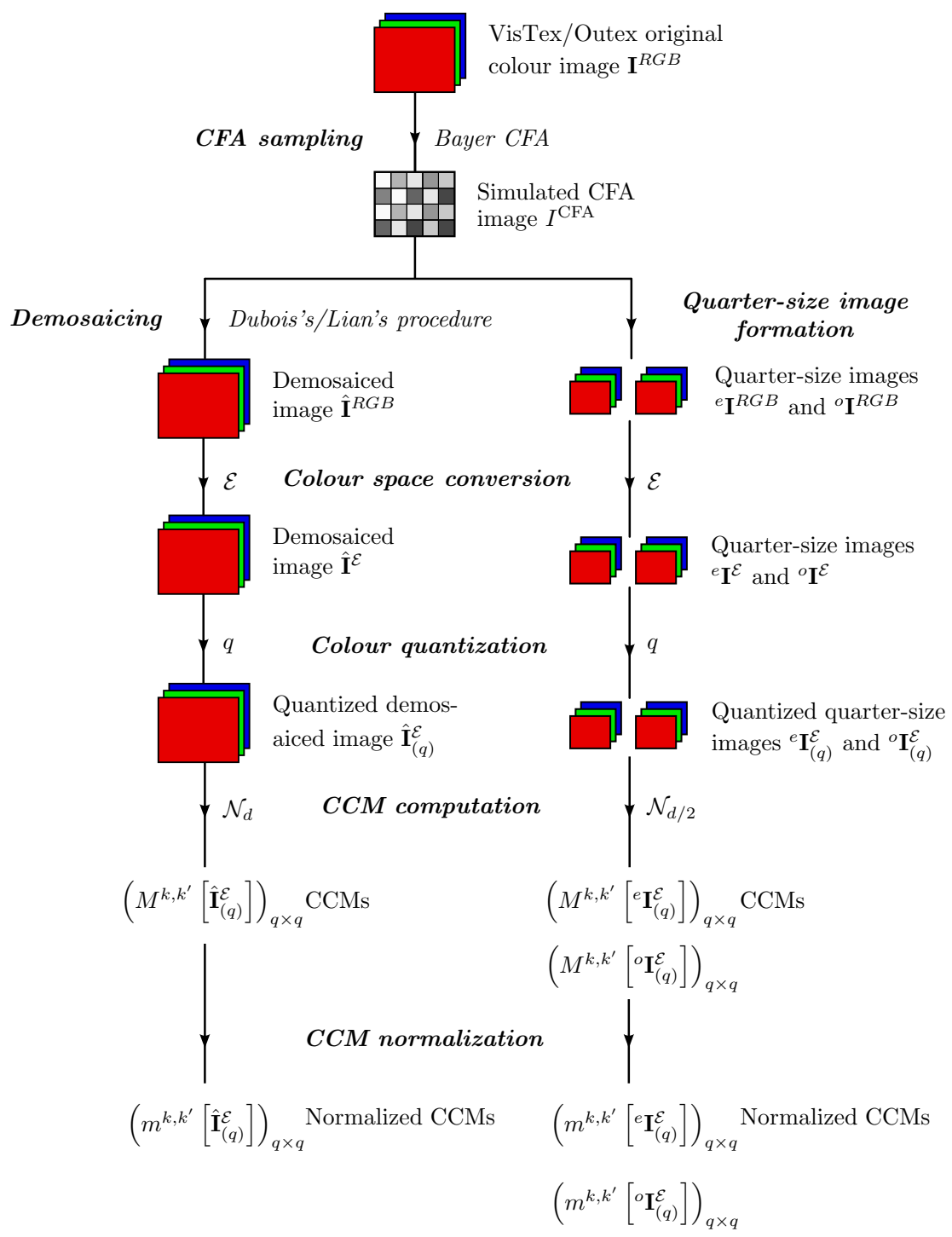

FIG. 7 Computation of the CCMs from the demosaiced colour image $\hat{\mathbf{I}}^{R G B}$ (left branch) and from the quarter-size image pair $\left({ }^{e} \mathbf{I}^{R G B},{ }^{o} \mathbf{I}^{R G B}\right)$ (right branch). 
ski et al. [33] have shown that a coarse quantization may be applied to CCMs before their relevance gets seriously affected: when $q$ is set to 32 or 64 , the analysis of such reduced-size matrices yields good classification results, while the processing time is reduced significantly. To study its influence, the parameter $q$ is set to the powers of 2 from 16 to 256 .

The numbers of columns $X$ and of rows $Y$ of $\hat{\mathbf{I}}_{(q)}^{\mathcal{E}}$ are twice as those of ${ }^{e} \mathbf{I}_{(q)}^{\mathcal{E}}$ and ${ }^{o} \mathbf{I}_{(q)}^{\mathcal{E}}$. To analyse textures with nearly the same spatial resolution for these different kinds of images, the neighbourhood distance parameter $d$ (see equation (2)) used in practice to compute the CCMs from $\hat{\mathbf{I}}_{(q)}^{\mathcal{E}}$ must be twice as that used to compute the CCMs from ${ }^{e} \mathbf{I}_{(q)}^{\mathcal{E}}$ and ${ }^{o} \mathbf{I}_{(q)}^{\mathcal{E}}$. Besides, due to minor changes on CCM fidelity whether $d$ is set to 2 or 8 (see table 1), this distance parameter is fixed to 2 to compute the CCMs from $\hat{\mathbf{I}}_{(q)}^{\mathcal{E}}$ (and to 1 to compute those from ${ }^{e} \mathbf{I}_{(q)}^{\mathcal{E}}$ and ${ }^{o} \mathbf{I}_{(q)}^{\mathcal{E}}$ ).

In short, two sets of CCMs are computed from the simulated CFA image:

- Six CCMs are extracted from the demosaiced colour image $\hat{\mathbf{I}}_{(q)}^{\mathcal{E}}$ (see subsection 2.2).

- Twelve CCMs are extracted from the quarter-size colour images ${ }^{e} \mathbf{I}_{(q)}^{\mathcal{E}}$ and ${ }^{o} \mathbf{I}_{(q)}^{\mathcal{E}}$ (see subsection 3.3).

\subsection{Selection of the pair of quarter-size images}

As explained in subsections 3.3 and 4.1, four different pairs of quarter-size images

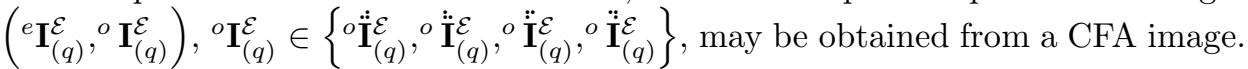
We here intend to find which pair provides the best classification results and should be retained for further experiments.

For each colour space $\mathcal{E}$ and each value of $q$, we apply a 1 -NN classifier to VisTex and Outex datasets using the four different pairs of quarter-size images. These pairs are then sorted according to their classification accuracies, and we associate a classification rank (ranging from 1 to 4 ) to each of them. To obtain the scores of classification performance of each pair according to each dataset and CCM similarity measure, we cumulate the classification ranks over the five values of $q$ previously considered, and over the two colour spaces considered for each dataset. A given pair is considered as the best one when the number of its first ranks is the highest (see table 2(a)). To assess the classification performance in depth, we also compare the total sums of the classification ranks, which have to be as low as possible (see table 2(b)). Each row in tables 2(a) and 2(b) corresponds to a dataset and a similarity measure, and its best result is written in bold face. The percentages to which these ranks correspond are not detailed here to ensure easy readability. But let us point out that they actually differ very slightly (by less than $0.75 \%$ ) among the four different pairs of quarter-size $\left({ }^{e} \mathbf{I}_{(q)}^{\mathcal{E}},{ }^{o} \mathbf{I}_{(q)}^{\mathcal{E}}\right),{ }^{o} \mathbf{I}_{(q)}^{\mathcal{E}} \in\left\{o \ddot{\mathbf{I}}_{(q)}^{\mathcal{E}},{ }^{o} \ddot{\mathbf{I}}_{(q)}^{\mathcal{E}}, o{ }^{\circ} \ddot{\mathbf{I}}_{(q)}^{\mathcal{E}},{ }^{o} \ddot{\mathbf{I}}_{(q)}^{\mathcal{E}}\right\}$, for given values of $q$ and $\mathcal{E}$. 
TABLE 2: Texture classification performance achieved by CCMs extracted from different pairs of quarter-size colour images $\left({ }^{e} \mathbf{I},{ }^{o} \mathbf{I}\right),{ }^{o} \mathbf{I} \in\left\{{ }^{o} \dot{\mathbf{I}},{ }^{o} \ddot{\mathbf{I}},{ }^{o} \ddot{\mathbf{I}}, o \ddot{\mathbf{I}}\right\}$. The distance parameter $d$ is set to 2 , and rank values are cumulated over $q=16,32,64,128$, and 256, and over the two colour spaces $\mathcal{E}$ considered for each dataset.

(a) Number of first classification ranks. The numbers in each row sum up to more than 10 (5 values of $q \times 2$ colour spaces) because several pairs equally perform best in many cases.

\begin{tabular}{|c|c||c|c|c|c|}
\hline Dataset & CCM similarity measure & ${ }^{\circ} \ddot{\mathbf{I}}$ & ${ }^{\circ} \ddot{\mathbf{I}}$ & ${ }^{\circ} \ddot{\mathbf{I}}$ & ${ }^{\circ} \ddot{\mathbf{I}}$ \\
\hline VisTex & Intersection & 5 & $\mathbf{8}$ & 6 & 6 \\
\cline { 2 - 6 } $\mathcal{E} \in\left\{R G B, I_{1} I_{2} I_{3}\right\}$ & Jeffrey divergence & 6 & $\mathbf{8}$ & $\mathbf{8}$ & 5 \\
\hline Outex & Intersection & $\mathbf{5}$ & 3 & 4 & 2 \\
\cline { 2 - 6 } $\mathcal{E} \in\{R G B, H S V\}$ & Jeffrey divergence & 2 & 5 & $\mathbf{6}$ & 3 \\
\hline Total number of first classification ranks & 18 & $\mathbf{2 4}$ & $\mathbf{2 4}$ & 16 \\
\hline
\end{tabular}

(b) Sum of the classification ranks.

\begin{tabular}{|c|c||c|c|c|c|}
\hline Dataset & CCM similarity measure & ${ }^{\circ} \dot{\mathbf{I}}$ & ${ }^{\circ} \dot{\mathbf{I}}$ & ${ }^{\circ} \mathbf{I}$ & ${ }^{\circ} \ddot{\mathbf{I}}$ \\
\hline VisTex & Intersection & 17 & $\mathbf{1 4}$ & 15 & 16 \\
\cline { 2 - 6 } $\mathcal{E} \in\left\{R G B, I_{1} I_{2} I_{3}\right\}$ & Jeffrey divergence & 16 & $\mathbf{1 2}$ & 14 & 19 \\
\hline Outex & Intersection & 20 & $\mathbf{1 8}$ & 21 & 26 \\
\cline { 2 - 6 } $\mathcal{E} \in\{R G B, H S V\}$ & Jeffrey divergence & 22 & 21 & $\mathbf{1 7}$ & 23 \\
\hline Total sum of classification ranks & 75 & $\mathbf{6 5}$ & 67 & 84 \\
\hline
\end{tabular}


Table 2(a) shows that the pairs $\left({ }^{e} \mathbf{I},{ }^{o} \dot{\overline{\mathbf{I}}}\right)$ and $\left({ }^{e} \mathbf{I},{ }^{o} \ddot{\mathbf{I}}\right)$ equally perform best the most times. Table 2(b) allows us to conclude that $\left({ }^{e} \mathbf{I},{ }^{o} \dot{\mathbf{I}}\right)$ is the best quarter-size image pair for texture classification, therefore retained hereafter. These results also confirm that other quarter-size images than ${ }^{o} \dot{\mathbf{I}} R G B$ may improve the classification performance since they contain less redundant information with ${ }^{e} \mathbf{I}^{R G B}$. This remains true when these images are converted to other colour spaces.

\subsection{Experimental results}

\subsubsection{Classification accuracy}

Tables 3(a) and 3(b) display the classification accuracies of the VisTex and Outex test images. For each similarity measure (intersection or Jeffrey divergence), the three columns contain the correct classification rates, according to each kind of images used to compute the CCMs: the (quantized and converted) demosaiced images $\hat{\mathbf{I}}_{(q)}^{\mathcal{E}}$ provided by two highly-efficient procedures (Dubois's [30] and Lian et al.'s [31]),

and the pair of quarter-size colour images $\left({ }^{e} \mathbf{I}_{(q)}^{\mathcal{E}},{ }^{o} \ddot{\mathbf{I}}_{(q)}^{\mathcal{E}}\right)$. For each value of $q$, each colour space $\mathcal{E}$, and each similarity measure, the best classification accuracy is written in bold face. To highlight the advantage of colour space conversion, we both display the results obtained with the original colour space $R G B$ and either $I_{1} I_{2} I_{3}$ or $H S V$.

By examining these tables, we first note that the rates of correctly classified images are very close to each other for the CCMs computed from the two kinds of images, whatever the similarity measure and the colour quantization level.

Except for one case (the VisTex dataset, when $q=64$ and with the intersection as similarity measure), the accuracy obtained with the colour space $I_{1} I_{2} I_{3}$ or $H S V$ is always higher than that obtained with $R G B$. This result shows that using a relevant colour space instead of $R G B$ improves the classification quality. It also highlights that extracting quarter-size colour images from the CFA image is an efficient solution, since this allows us to represent the colorimetric information of the CFA image in different colour spaces.

Tables 3(a) and 3(b) also show that CCMs extracted from the quarter-size colour images provide comparable (and even globally better) results with regard to CCMs extracted from the demosaiced images. Indeed, the resulting accuracy is higher or equal to that of the best demosaicing procedure (either Dubois's or Lian et al.'s) for 28 out of the 40 cases.

\subsubsection{Average match percentile}

Image retrieval consists in finding the images in a set that are the most similar to a test image. To achieve each retrieval, a set of $N_{t a r}$ target images is formed by picking one prototype image (the "true" image) from the same class as the 
TABLE 3: Correct classification rates (\%) of VisTex and Outex test images. The distance parameter $d$ is set to 2 .

(a) VisTex dataset ( $R G B$ and $I_{1} I_{2} I_{3}$ colour spaces).

\begin{tabular}{|c|c||c|c|c||c|c|c|}
\hline \multicolumn{2}{|l||}{} & \multicolumn{3}{c||}{ Intersection } & \multicolumn{3}{c|}{ Jeffrey divergence } \\
\multicolumn{2}{|l||}{} & \multicolumn{2}{|c||}{ CCMs from $\hat{\mathbf{I}}_{(q)}^{\mathcal{E}}$} & \multicolumn{2}{c|}{ CCMs from } & CCMs from $\hat{\mathbf{I}}_{(q)}^{\mathcal{E}}$ & CCMs from \\
& $\mathcal{E}$ & Dubois & Lian & $\left({ }^{e} \mathbf{I}_{(q)}^{\mathcal{E}},{ }^{o} \dot{\mathbf{I}}_{(q)}^{\mathcal{E}}\right)$ & Dubois & Lian & $\left({ }^{e} \mathbf{I}_{(q)}^{\mathcal{E}},{ }^{o} \dot{\mathbf{I}}_{(q)}^{\mathcal{E}}\right)$ \\
\hline \multirow{2}{*}{16} & $R G B$ & 96.99 & 96.99 & $\mathbf{9 7 . 6 9}$ & 97.22 & 97.22 & $\mathbf{9 7 . 4 5}$ \\
& $I_{1} I_{2} I_{3}$ & $\mathbf{9 8 . 1 5}$ & 97.92 & 97.92 & $\mathbf{9 8 . 8 4}$ & 98.61 & 98.15 \\
\hline \multirow{2}{*}{32} & $R G B$ & $\mathbf{9 7 . 6 9}$ & 97.22 & $\mathbf{9 7 . 6 9}$ & 97.22 & 97.45 & $\mathbf{9 7 . 9 2}$ \\
& $I_{1} I_{2} I_{3}$ & 97.92 & 98.38 & $\mathbf{9 8 . 6 1}$ & 98.38 & $\mathbf{9 8 . 6 1}$ & $\mathbf{9 8 . 6 1}$ \\
\hline \multirow{2}{*}{64} & $R G B$ & 97.22 & 97.22 & $\mathbf{9 8 . 3 8}$ & 97.45 & 97.45 & $\mathbf{9 8 . 3 8}$ \\
& $I_{1} I_{2} I_{3}$ & 97.92 & $\mathbf{9 8 . 1 5}$ & $\mathbf{9 8 . 1 5}$ & 98.38 & $\mathbf{9 8 . 6 1}$ & $\mathbf{9 8 . 6 1}$ \\
\hline \multirow{2}{*}{128} & $R G B$ & 97.22 & 96.99 & $\mathbf{9 8 . 1 5}$ & 97.45 & 97.69 & $\mathbf{9 8 . 3 8}$ \\
& $I_{1} I_{2} I_{3}$ & 98.61 & 98.38 & $\mathbf{9 8 . 8 4}$ & 98.61 & 98.61 & $\mathbf{9 8 . 8 4}$ \\
\hline \multirow{2}{*}{256} & $R G B$ & $\mathbf{9 7 . 2 2}$ & 96.99 & 94.91 & 96.30 & $\mathbf{9 6 . 7 6}$ & 92.36 \\
& $I_{1} I_{2} I_{3}$ & $\mathbf{9 8 . 8 4}$ & $\mathbf{9 8 . 8 4}$ & 98.61 & 98.61 & 98.61 & $\mathbf{9 8 . 8 4}$ \\
\hline
\end{tabular}

(b) Outex dataset ( $R G B$ and $H S V$ colour spaces).

\begin{tabular}{|c|c||c|c|c||c|c|c|}
\hline \multicolumn{2}{|l||}{} & \multicolumn{3}{c||}{ Intersection } & \multicolumn{3}{c|}{ Jeffrey divergence } \\
\multicolumn{1}{|l||}{} & CCMs from $\hat{\mathbf{I}}_{(q)}^{\mathcal{E}}$ & \multicolumn{2}{c|}{ CCMs from } & CCMs from $\hat{\mathbf{I}}_{(q)}^{\mathcal{E}}$ & CCMs from \\
& $\mathcal{E}$ & Dubois & Lian & $\left({ }^{e} \mathbf{I}_{(q)}^{\mathcal{E}},{ }^{o} \dot{\mathbf{I}}_{(q)}^{\mathcal{E}}\right)$ & Dubois & Lian & $\left({ }^{e} \mathbf{I}_{(q)}^{\mathcal{E}},{ }^{o} \dot{\mathbf{I}}_{(q)}^{\mathcal{E}}\right)$ \\
\hline \multirow{2}{*}{16} & $R G B$ & 91.47 & 91.47 & $\mathbf{9 1 . 6 2}$ & 92.50 & 92.21 & $\mathbf{9 2 . 7 9}$ \\
& $H S V$ & 92.35 & 92.06 & $\mathbf{9 2 . 7 9}$ & 93.82 & 93.97 & $\mathbf{9 4 . 4 1}$ \\
\hline \multirow{2}{*}{32} & $R G B$ & 92.65 & 92.79 & $\mathbf{9 3 . 5 3}$ & $\mathbf{9 3 . 3 8}$ & $\mathbf{9 3 . 3 8}$ & $\mathbf{9 3 . 3 8}$ \\
& $H S V$ & $\mathbf{9 4 . 2 6}$ & $\mathbf{9 4 . 2 6}$ & $\mathbf{9 4 . 2 6}$ & $\mathbf{9 5 . 0 0}$ & 94.41 & 94.71 \\
\hline \multirow{2}{*}{64} & $R G B$ & 92.94 & $\mathbf{9 3 . 3 8}$ & $\mathbf{9 3 . 3 8}$ & 93.53 & 93.09 & $\mathbf{9 3 . 9 7}$ \\
& $H S V$ & $\mathbf{9 4 . 7 1}$ & 94.56 & 94.56 & 94.71 & $\mathbf{9 5 . 0 0}$ & $\mathbf{9 5 . 0 0}$ \\
\hline \multirow{2}{*}{128} & $R G B$ & 93.82 & $\mathbf{9 4 . 2 6}$ & 93.97 & 93.38 & $\mathbf{9 3 . 5 3}$ & $\mathbf{9 3 . 5 3}$ \\
& $H S V$ & 94.71 & 94.85 & $\mathbf{9 5 . 0 0}$ & 94.85 & $\mathbf{9 5 . 0 0}$ & $\mathbf{9 5 . 0 0}$ \\
\hline \multirow{2}{*}{256} & $R G B$ & 93.09 & $\mathbf{9 3 . 3 8}$ & 89.85 & $\mathbf{9 3 . 0 9}$ & 92.94 & 87.50 \\
& $H S V$ & $\mathbf{9 5 . 1 5}$ & 94.71 & 94.12 & 94.85 & $\mathbf{9 5 . 1 5}$ & 94.12 \\
\hline
\end{tabular}


TABLE 4: AMP (\%) for VisTex and Outex datasets. The distance parameter $d$ is set to 2 .

(a) VisTex dataset ( $R G B$ and $I_{1} I_{2} I_{3}$ colour spaces).

\begin{tabular}{|c|c||c|c|c||c|c|c|}
\hline \multicolumn{2}{|l||}{} & \multicolumn{3}{c||}{ Intersection } & \multicolumn{3}{c|}{ Jeffrey divergence } \\
\multicolumn{2}{|l||}{} & \multicolumn{2}{|c||}{ CCMs from $\hat{\mathbf{I}}_{(q)}^{\mathcal{E}}$} & \multicolumn{2}{c|}{ CCMs from } & CCMs from $\hat{\mathbf{I}}_{(q)}^{\mathcal{E}}$ & CCMs from \\
& $\mathcal{E}$ & Dubois & Lian & $\left({ }^{e} \mathbf{I}_{(q)}^{\mathcal{E}},{ }^{o} \dot{\mathbf{I}}_{(q)}^{\mathcal{E}}\right)$ & Dubois & Lian & $\left({ }^{e} \mathbf{I}_{(q)}^{\mathcal{E}},{ }^{o} \dot{\mathbf{I}}_{(q)}^{\mathcal{E}}\right)$ \\
\hline \multirow{2}{*}{16} & $R G B$ & 94.89 & 94.92 & $\mathbf{9 5 . 3 2}$ & 95.23 & 95.27 & $\mathbf{9 5 . 6 3}$ \\
& $I_{1} I_{2} I_{3}$ & 96.50 & $\mathbf{9 6 . 5 8}$ & 96.22 & 96.84 & $\mathbf{9 6 . 9 3}$ & 96.81 \\
\hline \multirow{2}{*}{32} & $R G B$ & 95.30 & 95.34 & $\mathbf{9 5 . 7 6}$ & 95.60 & 95.65 & $\mathbf{9 6 . 0 4}$ \\
& $I_{1} I_{2} I_{3}$ & 96.85 & $\mathbf{9 6 . 9 7}$ & 96.80 & 97.14 & $\mathbf{9 7 . 2 4}$ & 97.13 \\
\hline \multirow{2}{*}{64} & $R G B$ & 95.42 & 95.48 & $\mathbf{9 5 . 9 8}$ & 95.70 & 95.75 & $\mathbf{9 6 . 1 9}$ \\
& $I_{1} I_{2} I_{3}$ & 96.89 & 96.97 & $\mathbf{9 7 . 0 9}$ & 97.18 & 97.25 & $\mathbf{9 7 . 3 6}$ \\
\hline \multirow{2}{*}{128} & $R G B$ & 95.55 & 95.60 & $\mathbf{9 6 . 3 2}$ & 95.77 & 95.82 & $\mathbf{9 6 . 4 1}$ \\
& $I_{1} I_{2} I_{3}$ & 96.96 & 97.02 & $\mathbf{9 7 . 2 4}$ & 97.10 & 97.19 & $\mathbf{9 7 . 4 4}$ \\
\hline \multirow{2}{*}{256} & $R G B$ & 95.75 & 95.80 & $\mathbf{9 6 . 6 8}$ & 95.85 & 95.89 & $\mathbf{9 6 . 3 9}$ \\
& $I_{1} I_{2} I_{3}$ & 96.92 & 96.99 & $\mathbf{9 7 . 2 6}$ & 97.06 & 97.15 & $\mathbf{9 7 . 4 3}$ \\
\hline
\end{tabular}

(b) Outex dataset ( $R G B$ and $H S V$ colour spaces).

\begin{tabular}{|c|c||c|c|c||c|c|c|}
\hline \multicolumn{2}{|l||}{} & \multicolumn{3}{c||}{ Intersection } & \multicolumn{3}{c|}{ Jeffrey divergence } \\
\multicolumn{1}{|l||}{} & \multicolumn{2}{|c|}{ CCMs from $\hat{\mathbf{I}}_{(q)}^{\mathcal{E}}$} & \multicolumn{2}{c|}{ CCMs from } & CCMs from $\hat{\mathbf{I}}_{(q)}^{\mathcal{E}}$ & CCMs from \\
& $\mathcal{E}$ & Dubois & Lian & $\left({ }^{e} \mathbf{I}_{(q)}^{\mathcal{E}},{ }^{o} \dot{\mathbf{I}}_{(q)}^{\mathcal{E}}\right)$ & Dubois & Lian & $\left({ }^{e} \mathbf{I}_{(q)}^{\mathcal{E}},{ }^{\circ} \dot{\mathbf{I}}_{(q)}^{\mathcal{E}}\right)$ \\
\hline \multirow{2}{*}{16} & $R G B$ & 97.17 & $\mathbf{9 7 . 1 8}$ & 97.02 & $\mathbf{9 7 . 3 7}$ & $\mathbf{9 7 . 3 7}$ & 97.08 \\
& $H S V$ & 98.42 & 98.43 & $\mathbf{9 8 . 6 5}$ & 98.53 & 98.53 & $\mathbf{9 8 . 7 1}$ \\
\hline \multirow{2}{*}{32} & $R G B$ & 97.22 & $\mathbf{9 7 . 2 3}$ & 97.17 & $\mathbf{9 7 . 3 6}$ & $\mathbf{9 7 . 3 6}$ & 97.30 \\
& $H S V$ & 98.66 & 98.68 & $\mathbf{9 8 . 8 0}$ & 98.69 & 98.71 & $\mathbf{9 8 . 8 2}$ \\
\hline \multirow{2}{*}{64} & $R G B$ & $\mathbf{9 7 . 2 1}$ & $\mathbf{9 7 . 2 1}$ & 97.18 & $\mathbf{9 7 . 3 2}$ & $\mathbf{9 7 . 3 2}$ & 97.29 \\
& $H S V$ & 98.70 & 98.71 & $\mathbf{9 8 . 8 3}$ & 98.70 & 98.71 & $\mathbf{9 8 . 8 3}$ \\
\hline \multirow{2}{*}{128} & $R G B$ & $\mathbf{9 7 . 2 0}$ & $\mathbf{9 7 . 2 0}$ & 97.19 & $\mathbf{9 7 . 3 1}$ & $\mathbf{9 7 . 3 1}$ & 97.28 \\
& $H S V$ & 98.68 & 98.70 & $\mathbf{9 8 . 8 2}$ & 98.68 & 98.70 & $\mathbf{9 8 . 8 2}$ \\
\hline \multirow{2}{*}{256} & $R G B$ & $\mathbf{9 7 . 2 1}$ & $\mathbf{9 7 . 2 1}$ & 97.20 & $\mathbf{9 7 . 3 0}$ & $\mathbf{9 7 . 3 0}$ & 97.21 \\
& $H S V$ & 98.66 & 98.69 & $\mathbf{9 8 . 7 9}$ & 98.65 & 98.67 & $\mathbf{9 8 . 7 3}$ \\
\hline
\end{tabular}


test image, plus all the prototypes of all the other classes. Then, we compute the similarity to the test image of each of the $N_{t a r}$ target images. The position of the true image in the sorted list of similarity values is called the rank $r$. The match percentile, defined in percentage as $M P=100 \times \frac{N_{t a r}-r}{N_{t a r}-1}$, is an indicator of the retrieval performance. As expected, $M P$ reaches $100 \%$ when the true image is ranked first (perfect retrieval); a value of $99 \%$ means that the true image is more similar to the test image than $99 \%$ of the other target images. The global retrieval performance is assessed by the Average Match Percentile $(A M P)$ [34] over all possible true images for a given test image and over all test images in the dataset.

Tables 4(a) and 4(b) display the average match percentile obtained with VisTex and Outex datasets. In all cases, the $A M P$ provided with the relevant colour space is higher than that provided with the $R G B$ colour space. This result confirms that coding the colours in a relevant colour space improves the quality of texture retrieval. Besides, the $A M P$ provided by CCMs computed from quarter-size images is higher than, or equal to that provided by CCMs from demosaiced images for 26 out of the 40 cases. Therefore, we can conclude that the texture retrieval performance achieved by quarter-size images formed from the CFA image is comparable to that of demosaiced images. This sharpens and confirms classification results.

\subsubsection{Result summary}

Table 5(a) summarizes the classification and retrieval results presented in tables 3(a)-4(b). It allows the reader to easily compare the performance of the two schemes, either CCMs extracted from the demosaiced image $\hat{\mathbf{I}}_{(q)}^{\mathcal{E}}$ or from the quarter-size image pair $\left({ }^{e} \mathbf{I}_{(q)}^{\mathcal{E}},{ }^{o} \ddot{\dot{\mathbf{I}}_{(q)}^{\mathcal{E}}}\right)$. To give a synthetic view, the number of times that each scheme performs best, for a given problem, dataset, and similarity measure, are cumulated over the five values of the quantization parameter $q$ and over the two colour spaces $\mathcal{E}$ considered for the dataset.

Each class is represented by 16 images in VisTex and 20 images in Outex dataset. A statistical bias could result from the given split of these images into prototype and test sets during the holdout procedure. To avoid it, we also evaluate classification accuracies with different prototype and test sets. The available images for each class is divided into 4 subsets of 4 (VisTex) and 5 (Outex) images. Two subsets are used as prototypes, and two as test images, which yields $C(4,2)=6$ different combinations. The same 6 combinations of prototype and test images are used for the retrieval problem. While tables $3(\mathrm{a})-5$ (a) are based on the holdout defined in the original Contrib-TC-00006 and Outex-TC-00013 datasets, table 5(b) summarizes classification and retrieval performances using all 6 combinations.

The results in table 5(b) are consistent with those in table 5(a). The $(6$ times) higher number of cases noticeably highlights that Lian et al.'s demosaicing procedure is less detrimental to textural information than Dubois's, although intersection-based CCM similarities to the original colour image are very close (see table 1). But these results mainly confirm that the proposed scheme outperforms classical CCM-based approaches using demosaiced images for texture classification 
TABLE 5: Summary of the scheme performances in classification and retrieval problems, given by the number of times that each scheme performs best. The distance parameter $d$ is set to 2 . The ranks are cumulated over $q=16,32,64,128$, and 256, and over the two colour spaces considered for each dataset (a), and also over the 6 subset combinations of prototype and test images (b). The total numbers of first ranks sum up to more than the 40 (a) and the 240 (b) cases for each problem, since the two schemes may equally perform best.

(a) Using the same prototype and test images as for tables $3(\mathrm{a})-4(\mathrm{~b})$.

\begin{tabular}{|c|c|c|c|c|c|}
\hline $\begin{array}{l}\text { Problem (per- } \\
\text { form. measure) }\end{array}$ & Dataset & $\begin{array}{c}\text { CCM similarity } \\
\text { measure }\end{array}$ & $\begin{array}{l}\text { CCMs f } \\
\text { Dubois }\end{array}$ & $\begin{array}{l}\mathrm{m} \hat{\mathbf{I}}_{(q)}^{\mathcal{E}} \\
\text { Lian }\end{array}$ & 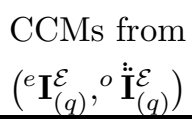 \\
\hline \multirow{4}{*}{$\begin{array}{l}\text { Classification } \\
\text { (correct } \\
\text { classification } \\
\text { rate) }\end{array}$} & \multirow{2}{*}{$\begin{array}{c}\text { VisTex } \\
\mathcal{E} \in\left\{R G B, I_{1} I_{2} I_{3}\right\}\end{array}$} & Intersection & 4 & 2 & 7 \\
\hline & & Jeffrey divergence & 1 & 3 & 8 \\
\hline & \multirow{2}{*}{$\begin{array}{c}\text { Outex } \\
\mathcal{E} \in\{R G B, H S V\}\end{array}$} & Intersection & 3 & 4 & 6 \\
\hline & & Jeffrey divergence & 3 & 5 & 7 \\
\hline \multicolumn{3}{|c|}{ Total number of first classification ranks } & 11 & 14 & 28 \\
\hline \multirow{4}{*}{$\begin{array}{c}\text { Retrieval } \\
(A M P)\end{array}$} & \multirow{2}{*}{$\begin{array}{c}\text { VisTex } \\
\mathcal{E} \in\left\{R G B, I_{1} I_{2} I_{3}\right\}\end{array}$} & Intersection & 0 & 2 & 8 \\
\hline & & Jeffrey divergence & 0 & 2 & 8 \\
\hline & \multirow{2}{*}{$\begin{array}{c}\text { Outex } \\
\mathcal{E} \in\{R G B, H S V\}\end{array}$} & Intersection & 3 & 5 & 5 \\
\hline & & Jeffrey divergence & 5 & 5 & 5 \\
\hline \multicolumn{3}{|c|}{ Total number of first retrieval ranks } & 8 & 14 & 26 \\
\hline \multicolumn{3}{|c|}{ 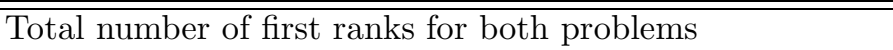 } & $\overline{\overline{19}}$ & $\overline{28}$ & $\overline{54}$ \\
\hline
\end{tabular}

(b) Using 6 different prototype and test image subsets.

\begin{tabular}{|c|c|c|c|c|c|}
\hline $\begin{array}{l}\text { Problem (per- } \\
\text { form. measure) }\end{array}$ & Dataset & $\begin{array}{l}\text { CCM similarity } \\
\text { measure }\end{array}$ & $\begin{array}{l}\text { CCMs f } \\
\text { Dubois }\end{array}$ & $\begin{array}{l}\mathrm{m} \hat{\mathbf{I}}_{(q)}^{\mathcal{E}} \\
\operatorname{Lian}\end{array}$ & $\begin{array}{l}\text { CCMs from } \\
\left({ }^{e} \mathbf{I}_{(q)}^{\mathcal{E}},{ }^{o} \ddot{\mathbf{I}}_{(q)}^{\mathcal{E}}\right)\end{array}$ \\
\hline \multirow{4}{*}{$\begin{array}{l}\text { Classification } \\
\text { (correct } \\
\text { classification } \\
\text { rate) }\end{array}$} & \multirow{2}{*}{$\begin{array}{c}\text { VisTex } \\
\mathcal{E} \in\left\{R G B, I_{1} I_{2} I_{3}\right\}\end{array}$} & Intersection & 12 & 21 & 42 \\
\hline & & Jeffrey divergence & 11 & 21 & 39 \\
\hline & \multirow{2}{*}{$\begin{array}{c}\text { Outex } \\
\mathcal{E} \in\{R G B, H S V\}\end{array}$} & Intersection & 20 & 25 & 24 \\
\hline & & Jeffrey divergence & 22 & 27 & 25 \\
\hline \multicolumn{3}{|c|}{ Total number of first classification ranks } & 65 & 94 & 130 \\
\hline \multirow{4}{*}{$\begin{array}{c}\text { Retrieval } \\
(A M P)\end{array}$} & \multirow{2}{*}{$\begin{array}{c}\text { VisTex } \\
\mathcal{E} \in\left\{R G B, I_{1} I_{2} I_{3}\right\}\end{array}$} & Intersection & 0 & 13 & $\overline{47}$ \\
\hline & & Jeffrey divergence & 0 & 12 & 48 \\
\hline & \multirow{2}{*}{$\begin{array}{c}\text { Outex } \\
\mathcal{E} \in\{R G B, H S V\}\end{array}$} & Intersection & 16 & 27 & 31 \\
\hline & & Jeffrey divergence & 19 & 24 & 30 \\
\hline \multicolumn{3}{|c|}{ Total number of first retrieval ranks } & 35 & 76 & 156 \\
\hline \multicolumn{3}{|c|}{ Total number of first ranks for both problems } & 100 & 170 & 286 \\
\hline
\end{tabular}


TABLE 6: Numbers of elementary operations (top rows) and average processing times (bottom rows) required to compute the CCMs.

\begin{tabular}{|l|c|c|c|}
\hline & \multicolumn{2}{|c|}{ CCMs from $\hat{\mathbf{I}}_{(q)}^{\mathcal{E}}$} & CCMs from \\
Processing step & Dubois & $\operatorname{Lian}$ & $\left({ }^{e} \mathbf{I}_{(q)}^{\mathcal{E}},{ }^{o} \dot{\mathbf{I}}_{(q)}^{\mathcal{E}}\right)$ \\
\hline 1a. Demosaicing & $145 X Y$ & $65 X Y$ & 0 \\
\hline $\begin{array}{l}\text { 1b. Quarter-size image } \\
\text { formation }\end{array}$ & 0 & 0 & $X Y$ \\
\hline 2. Colour space conversion & $15 X Y$ & $15 X Y$ & $2 \times 15 \frac{X}{2} \frac{Y}{2}$ \\
\hline 3. Colour quantization & $3 X Y$ & $3 X Y$ & $2 \times 3 \frac{X}{2} \frac{Y}{2}$ \\
\hline 4. CCM computation & $48 X Y$ & $48 X Y$ & $2 \times 48 \frac{X}{2} \frac{Y}{2}$ \\
\hline 5. CCM normalization & $12 q^{2}$ & $12 q^{2}$ & $2 \times 12 q^{2}$ \\
\hline $\begin{array}{l}\text { Total number of } \\
\text { elementary operations }\end{array}$ & $211 X Y+12 q^{2}$ & $131 X Y+12 q^{2}$ & $34 X Y+24 q^{2}$ \\
\hline \hline Demosaicing (1a) & $64 \mathrm{~ms}$ & $42 \mathrm{~ms}$ & $0 \mathrm{~ms}$ \\
\hline Other processing steps (1b-5) & $19 \mathrm{~ms}$ & $19 \mathrm{~ms}$ & $14 \mathrm{~ms}$ \\
\hline $\begin{array}{l}\text { Total average } \\
\text { processing time }\end{array}$ & $83 \mathrm{~ms}$ & $61 \mathrm{~ms}$ & $14 \mathrm{~ms}$ \\
\hline
\end{tabular}

and retrieval. The next subsection shows that the proposed scheme using quartersize images is fully relevant as far as computational complexity is considered.

\subsection{Computational complexity}

To complete this experimental section, we propose to compare the computational complexity required to compare two texture images when using either the quarter-size image pairs or demosaiced images. This study is based on the number of elementary operations required by the compared schemes and on their processing time. The sole elementary operations considered here are arithmetic operations, all being equally weighted in the complexity estimation. Array indexing (or memory access) associated with each elementary operation is not taken into account.

\subsubsection{CCM computation}

Table 6 summarizes the number of elementary operations required to compute the CCMs from a CFA image $I^{\mathrm{CFA}}$ of size $X \times Y$ pixels, depending on the tested 
scheme: we either estimate the demosaiced image $\hat{\mathbf{I}}^{R G B}$ (classical approach) or form the pair of quarter-size images (proposed strategy). Then, we perform the colour space conversion and level quantization before normalized CCMs can be computed.

Dubois's demosaicing procedure is based on Alleysson et al.'s approach using frequency selection [35], and is assumed to be similarly complex. It is not easy to evaluate the computational complexity of such sophisticated algorithms, but Lian et al. have discussed this topic for several ones [31]. These authors indicate that $145 X Y$ elementary operations are required by the frequency-selection method, and $65 X Y$ operations for their own procedure.

Since the $(R, G, B)$ colour components are coded into most of colour spaces thanks to a linear transformation [19], we retain the corresponding number of elementary operations as that of the colour conversion step. It amounts to 3 multiplications and 2 additions for each colour component, requiring a total of 15 operations by pixel. Colour space conversion hence requires $15 X Y$ elementary operations for the demosaiced image, and only half that number for the two quarter-size images of size $\frac{X}{2} \times \frac{Y}{2}$ pixels.

The quantization step of the three colour components requires $3 X Y$ elementary operations for the demosaiced image, and only half that number for the two quartersize images.

Because array indexing is ignored here, the number of elementary operations required to compute a CCM only depends on the number of co-occurrences taken into account at each pixel. Since the neighbourhood used to compute the CCMs contains 8 pixels, the number of co-occurrences amounts to $48 X Y$ for the $6 \mathrm{CCMs}$ computed from a demosaiced image, and only half that number for the $12 \mathrm{CCMs}$ computed from the pair of quarter-size images.

Normalizing one CCM requires $q^{2}-1$ additions and $q^{2}$ divisions, hence about $2 q^{2}$ operations. So, the normalization step requires about $12 q^{2}$ operations for the 6 CCMs extracted from the demosaiced image, and twice that number for the 12 CCMs extracted from the pair of quarter-size images.

Overall, the number of elementary operations required to compute the CCMs extracted from the demosaiced colour image reaches at least $131 X Y+12 q^{2}$, whereas that required to compute the CCMs from the pair of quarter-size images is $34 X Y+$ $24 q^{2}$.

For our experiments, the demosaicing procedures are available as Matlab code [30, 31], whereas we implemented other steps (including quarter-size image formation) as Java plugins under ImageJ [36]. Hence, we finally give a rough idea of the processing times required by these operations (see last three rows of table 6 ). The processing times, obtained on a Core ${ }^{\mathrm{TM}_{2}}$ Duo PC at $1.58 \mathrm{GHz}$ with 4 Go RAM, are averaged over 100 images of each dataset and five values of $q$. They show that demosaicing is a time-consuming step that should be avoided, and that CCMs alone are computed faster with our scheme. 


\subsubsection{CCM comparison}

Measuring the similarity between two CCMs thanks to the intersection (see equation (4)) requires $q^{2}$ minimums and $q^{2}-1$ additions, hence about $2 q^{2}$ operations. So, texture comparison requires $12 q^{2}$ operations using the CCMs extracted from the demosaiced image, and $24 q^{2}$ operations using the CCMs extracted from the pair of quarter-size images.

Overall, comparing two colour texture images is divided into two successive steps: once the CCMs have been computed, they are compared thanks to the intersection similarity measure. When the CCMs are computed from demosaiced colour images, performing this comparison requires at least $2 \times\left(131 X Y+12 q^{2}\right)+$ $12 q^{2}=262 X Y+36 q^{2}$ operations to compute the CCMs and compare them. When the CCMs are computed from pairs of quarter-size images, the texture comparison requires $2 \times\left(34 X Y+24 q^{2}\right)+24 q^{2}=68 X Y+72 q^{2}$ operations. Assuming for simplicity that the value of $q^{2}$ is of the same order of magnitude as $X Y$, our texture comparison strategy requires nearly twice fewer elementary operations. The average processing times in the worst case $q=256$ (131 $\mathrm{ms}$ for CCMs from demosaiced images and $42 \mathrm{~ms}$ for our scheme) are even in a ratio above $3: 1$.

\section{CONCLUSION}

This paper focuses on colour texture classification from images delivered by single-sensor colour cameras. Such devices actually acquire CFA images, in which each pixel is characterized by only one of the three colour components, and from which colour images have to be estimated. We experimentally show that the demosaicing step, which estimates the two missing colour components at each pixel to deliver the demosaiced colour images, alters the texture representation quality and is time-consuming.

This leads us to develop an original approach that extracts texture descriptors using CFA images without any colour estimation. For this purpose, we propose to form a pair of quarter-size colour images directly from any CFA image. Although the size of these quarter-size images is reduced, they do not result from any colour estimation (unlike demosaiced images), and allow us to code colours in various colour spaces (unlike CFA images). Standard texture features can then be computed from these colour images, and used to compare textures in a classification scheme. Here, we choose the chromatic co-occurrence matrices that provide satisfying performances.

Experimental results from benchmark texture datasets show that better classification accuracies and recognition rates are reached by comparing CCMs extracted from pairs of quarter-size images rather than CCMs extracted from demosaiced colour images. Moreover, our new approach is less time-consuming than that based on demosaiced images, while still taking advantage of the properties of colour spaces. Such outstanding performance could be exploited whenever a texture classification scheme analyses images acquired by single-sensor colour cameras. 
This study introduces a new low-level strategy of CFA image analysis that provides promising results. It highlights the fact that demosaicing may not be useful and should even be avoided to analyse CFA images. Because most colour cameras actually deliver CFA images, such an approach could be used in every application achieving texture analysis. Fast colour-texture segmentation is required for instance by on-board low-level image analysis algorithms running in real time, such as for mobile robots control [37] or road detection in vehicle environment [38]. But CCMs are known to be sensitive to illumination variations [39], and CCMbased texture classification only provides satisfying results when the illumination is constant. This is a key issue in the analysis of outdoor scene images. To deal with it, we now intend to extract texture descriptors from the CFA image that are as invariant as possible to illumination changes.

\section{REFERENCES}

[1] Martínez-Alajarín, J., Luis-Delgado, J. D., and Tomás-Balibrea, L. M.: Automatic system for quality-based classification of marble textures, IEEE Transactions on Systems, Man and Cybernetics - Part C, 2005, 35, (4), pp. 488-497.

[2] Busin, L., Vandenbroucke, N., Macaire, L., Postaire, J.-G., and Tahon, P.: Flaw detection on decorated glasses by color image processing. Proc. 8th Int. Conf. Quality Control by Artificial Vision (QCAV'07), Vol. 6356, SPIE, Le Creusot, France, May 2007, p. 63560F.

[3] Akhloufi, M. A., Maldague, X., and Ben Larbi, W.: A new color-texture approach for industrial products inspection, Journal of Multimedia, 2008, 3, (3), pp. $44-50$.

[4] Kim, D. G., Burks, T. F., Qin, J., and Bulanon, D. M.: Classification of grapefruit peel diseases using color texture feature analysis, International Journal of Agricultural and Biological Engineering, 2009, 2, (3), pp. 41-50.

[5] Sertel, O., Kong, J., Çatalyürek, U. V., Lozanski, G., Saltz, J. H., and Gurcan, M. N.: Histopathological image analysis using model-based intermediate representations and color texture: follicular lymphoma grading, Journal of Signal Processing Systems, 2009, 55, (1-3), pp. 169-183.

[6] Li, Z., Liu, Y., Hayward, R., and Walker, R.: Color and texture feature fusion using kernel PCA with application to object-based vegetation species classification. Proc. Int. Conf. Image Processing (ICIP'10), Hong Kong, China, Sep. 2010, pp. 2701-2704.

[7] Münzenmayer, C., Volk, H., Küblbeck, C., Spinnler, K., and Wittenberg, T.: Multispectral texture analysis using interplane sum- and difference-histograms. Proc. 24th DAGM Symp. Pattern Recognition, Vol. 2449 of Lecture Notes in Computer Science, Zürich, Switzerland, Sep. 2002, pp. 42-49. 
[8] El Maia, H., Hammouch, A., and Aboutajdine, D.: Color-texture analysis by mutual information for multispectral image classification. Proc. Pacific Rim Conf. Communications, Computers and Signal Processing (PacRim'09), Victoria, BC, Canada, Aug. 2009, pp. 359-364.

[9] Ojala, T., Pietikäinen, M., and Mäenpää, T.: Multiresolution gray-scale and rotation invariant texture classification with local binary patterns, IEEE Transactions on Pattern Analysis and Machine Intelligence, 2002, 24, (7), pp. 971987.

[10] Haralick, R.: Statistical and structural approaches to texture, IEEE Transactions on Systems, Man and Cybernetics, 1979, 67, (5), pp. 786-804.

[11] Palm, C.: Color texture classification by integrative co-occurrence matrices, Pattern Recognition, 2004, 37, (5), pp. 965-976.

[12] Zheng, C., Sun, D.-W., and Zheng, L.: A new region-primitive method for classification of colour meat image texture based on size, orientation and contrast, Meat Science, 2007, 76, (4), pp. 620-627.

[13] Qazi, I.-U.-H., Alata, O., Burie, J.-C., Moussa, A., and Fernandez-Maloigne, C.: Choice of a pertinent color space for color texture characterization using parametric spectral analysis, Pattern Recognition, 2011, 44, (1), pp. 16-31.

[14] Porebski, A., Vandenbroucke, N., and Macaire, L.: A multi color space approach for texture classification: experiments with Outex, Vistex and Barktex image databases. Proc. 5th Eur. Conf. Colour in Graphics, Image and Vision (CGIV'10), Joensuu, Finland, June 2010, pp. 314-319.

[15] Losson, O., Macaire, L., and Yang, Y.: Comparison of color demosaicing methods, Advances in Imaging and Electron Physics, 2010, 162, pp. 173-265.

[16] Tamburrino, D., Alleysson, D., Meylan, L., and Süsstrunk, S.: Digital camera workflow for high dynamic range images using a model of retinal processing. Proc. 20th IS\&T/SPIE Electronic Imaging Annual Symp.: Digital Photography IV, Vol. 6817, San Jose, CA, USA, Jan. 2008, pp. 68170J1-12.

[17] Zhang, L., Lukac, R., Wu, X., and Zhang, D.: PCA-based spatially adaptive denoising of CFA images for single-sensor digital cameras, IEEE Transactions on Image Processing, 2009, 18, (4), pp. 797-812.

[18] Condat, L.: A simple, fast and efficient approach to denoisaicking: Joint demosaicking and denoising. Proc. Int. Conf. Image Processing (ICIP'10), Hong Kong, China, Sep. 2010, pp. 905-908.

[19] Busin, L., Vandenbroucke, N., and Macaire, L.: Color spaces and image segmentation, Advances in Imaging and Electron Physics, 2008, 151, (2), pp. $65-168$. 
[20] Rosenfeld, A.: Multispectral texture, IEEE Transactions on Systems, Man and Cybernetics, 1982, 12, (1), pp. 79-84.

[21] Porebski, A., Vandenbroucke, N., and Macaire, L.: Iterative feature selection for color texture classification. Proc. Int. Conf. Image Processing (ICIP'07), Vol. 3, San Antonio, Texas, USA, Sep. 2007, pp. 509-512.

[22] Rubner, Y., Puzicha, J., Tomasi, C., and Buhmann, J. M.: Empirical evaluation of dissimilarity measures for color and texture, Computer Vision and Image Understanding, 2001, 84, (1), pp. 25-43.

[23] Lategahn, H., Gross, S., Stehle, T., and Aach, T.: Texture classification by modeling joint distributions of local patterns with gaussian mixtures, IEEE Transactions on Image Processing, 2010, 19, (6), pp. 1548-1557.

[24] Hu, R., Rüger, S., Song, D., Liu, H., and Huang, Z.: Comparing dissimilarity measures for content-based image retrieval. Proc. Int. Conf. Multimedia and Expo (ICME'08), Hannover, Germany, June 2008, pp. 1365-1368.

[25] Ma, Y., Gu, X., and Wang, Y.: Histogram similarity measure using variable bin size distance, Computer Vision and Image Understanding, 2010, 114, (8), pp. 981-989.

[26] Mäenpää, T., and Pietikäinen, M.: Classification with color and texture: jointly or separately?, Pattern Recognition, 2004, 37, (8), pp. 1629-1640.

[27] Muselet, D., Macaire, L., and Postaire, J.-G.: A new approach for color person image indexing and retrieval, Machine Graphics \& Vision, 2002, 11, (2/3), pp. $257-283$.

[28] Li, X., Gunturk, B. K., and Zhang, L.: Image demosaicing: a systematic survey. Proc. Int. Conf. Visual Communications and Image Processing (VCIP'08), Vol. 6822, San Jose, California, USA, Jan. 2008, pp. 68221J1-15.

[29] Picard, R., Graczyk, C., Mann, S., Wachman, J., Picard, L., and Campbell, L.: Vision texture database (VisTex), 1995.

URL http://vismod.media.mit.edu/pub/VisTex/VisTex.tar.gz, accessed June 2012.

[30] Dubois, E.: Frequency-domain methods for demosaicking of Bayer-sampled color images, IEEE Signal Processing Letters, 2005, 12, (12), pp. 847-850.

URL http://lcavwww.epfl.ch/reproducible_research/ VandewalleKAS07, accessed June 2012.

[31] Lian, N.-X., Chang, L., Tan, Y.-P., and Zagorodnov, V.: Adaptive filtering for color filter array demosaicking, IEEE Transactions on Image Processing, 2007, 16, (10), pp. 2515-2525.

URL http://en.pudn.com/downloads142/sourcecode/graph/texture_ mapping/detail619008_en.html, accessed June 2012. 
[32] Ojala, T., Mäenpää, T., Pietikäinen, M., Viertola, J., Kyllönen, J., and Huovinen, S.: Outex new framework for empirical evaluation of texture analysis algorithms. Proc. 16th Int. Conf. Pattern Recognition (ICPR'02), Vol. 1, 2002, pp. 701-706.

URL http://www.outex.oulu.fi/temp/, accessed June 2012.

[33] Porebski, A., Vandenbroucke, N., and Macaire, L.: Selection of color texture features from reduced size chromatic co-occurrence matrices. Proc. Int. Conf. Signal and Image Processing Applications (ICSIPA'09), Kuala Lumpur, Malaysia, Nov. 2009, pp. 273-278.

[34] Swain, M. J., and Ballard, D. H.: Color indexing, International Journal of Computer Vision, 1991, 7, (1), pp. 11-32.

[35] Alleysson, D., Süsstrunk, S., and Hérault, J.: Linear demosaicing inspired by the human visual system, IEEE Transactions on Image Processing, 2005, 14, (4), pp. 439-449.

[36] Rasband, W. S.: ImageJ, U. S. National Institutes of Health, Bethesda, MD, USA, 1997-2011.

URL http://imagej.nih.gov/ij/, accessed June 2012.

[37] Blas, M. R., Agrawal, M., Sundaresan, A., and Konolige, K.: Fast color/texture segmentation for outdoor robots. Proc. Int. Conf. Intelligent Robots and Systems (IROS 2008), Nice, France, Sep. 2008, pp. $4078-4085$.

[38] Kang, Y., Kidono, K., Kimura, Y., and Ninomiya, Y.: Texture-based objects recognition for vehicle environment perception using a multiband camera, Advances in Visual Computing, Vol. 4842 of Lecture Notes in Computer Science, Springer, 2007, pp. 582-591.

[39] Muselet, D., and Macaire, L.: Combining color and spatial information for object recognition across illumination changes, Pattern Recognition Letters, 2007, 28, pp. 1176-1185. 